TRANSACTIONS OF THE

AMERICAN MATHEMATICAL SOCIETY

Volume 363, Number 4, April 2011, Pages 1821-1854

S 0002-9947(2010)05147-5

Article electronically published on November 8, 2010

\title{
UNIVERSAL BOUNDS FOR EIGENVALUES OF THE POLYHARMONIC OPERATORS
}

\author{
JÜRGEN JOST, XIANQING LI-JOST, QIAOLING WANG, AND CHANGYU XIA
}

\begin{abstract}
We study eigenvalues of polyharmonic operators on compact Riemannian manifolds with boundary (possibly empty). In particular, we prove a universal inequality for the eigenvalues of the polyharmonic operators on compact domains in a Euclidean space. This inequality controls the $k$ th eigenvalue by the lower eigenvalues, independently of the particular geometry of the domain. Our inequality is sharper than the known Payne-Pólya-Weinberg type inequality and also covers the important Yang inequality on eigenvalues of the Dirichlet Laplacian. We also prove universal inequalities for the lower order eigenvalues of the polyharmonic operator on compact domains in a Euclidean space which in the case of the biharmonic operator and the buckling problem strengthen the estimates obtained by Ashbaugh. Finally, we prove universal inequalities for eigenvalues of polyharmonic operators of any order on compact domains in the sphere.
\end{abstract}

\section{INTRODUCTION}

Let $\Omega$ be a connected bounded domain with smooth boundary in an $n(\geq 2)$ dimensional Euclidean space $\mathbb{R}^{n}$ and let $\nu$ be the outward unit normal vector field of $\partial \Omega$. Denote by $\Delta$ the Laplace operator on $\mathbb{R}^{n}$ and let $l$ be a positive integer. Solutions of $\Delta u=0$ on a domain $\Omega \subset \mathbb{R}^{n}$ are of course the classical harmonic functions which describe the equilibrium position of an elastic homogeneous membrane. Solutions of $\Delta^{2} u=0$ are called biharmonic, and they model equilibria of homogeneous plates. Similarly, solutions of $\Delta^{l} u=0, l \in \mathbb{N}$, are called polyharmonic.

One then naturally considers the eigenvalue problem

$$
\begin{aligned}
& (-\Delta)^{l} u=\lambda u \quad \text { in } \Omega \\
& \left.u\right|_{\partial \Omega}=\left.\frac{\partial u}{\partial \nu}\right|_{\partial \Omega}=\cdots=\left.\frac{\partial^{l-1} u}{\partial \nu^{l-1}}\right|_{\partial \Omega}=0 .
\end{aligned}
$$

Let

$$
0<\lambda_{1} \leq \lambda_{2} \leq \lambda_{3} \leq \cdots
$$

denote the successive eigenvalues, where each eigenvalue is repeated according to its multiplicity.

The case $l=1$ has of course been well-studied, beginning with the work of Weyl We and Courant-Hilbert $\mathrm{CH}$. But also for $l \geq 2$, polyharmonic functions have

Received by the editors August 19, 2008.

2010 Mathematics Subject Classification. Primary 35P15, 53C20.

Key words and phrases. Universal bounds, eigenvalues, polyharmonic operator, Riemannian manifolds, Euclidean space, spheres. 
interesting applications in physics. For example, the Airy function in mechanics is a bi-harmonic function. More generally, a clamped plate in equilibrium is a solution of the bi-harmonic problem

$$
\begin{aligned}
& (-\Delta)^{2} v=0 \quad \text { in } \Omega \\
& \left.v\right|_{\partial \Omega}=\left.\frac{\partial v}{\partial \nu}\right|_{\partial \Omega}=0
\end{aligned}
$$

in a two-dimensional $\Omega$. An oscillating clamped plate then satisfies

$$
\begin{aligned}
& (-\Delta)^{2} v+v_{t t}=0 \quad \text { in } \Omega, t \geq 0, \\
& \left.v\right|_{\partial \Omega}=\left.\frac{\partial v}{\partial \nu}\right|_{\partial \Omega}=0
\end{aligned}
$$

and a separation of variables $v(x, y, t)=u(x, y) g(t)$ leads to the eigenvalue problem

$$
\begin{aligned}
& (-\Delta)^{2} u=\lambda u \quad \text { in } \Omega, \\
& \left.u\right|_{\partial \Omega}=\left.\frac{\partial u}{\partial \nu}\right|_{\partial \Omega}=0 .
\end{aligned}
$$

This problem has already been studied by Courant [Co. He derived the Weyl type law

$$
\lambda_{k} \sim\left(\frac{4 \pi k}{\operatorname{area}(\Omega)}\right)^{2} .
$$

In this paper, we investigate the eigenvalues of the problem (1.1) for general $l$. We are interested in so-called universal properties, that is, properties that do not depend on the specific domain $\Omega$, but only on its dimension $n$. These universal properties then take the form of relations between different eigenvalues. Naturally, the first eigenvalue $\lambda_{1}$ plays a distinguished role. Since this eigenvalue can often be estimated in terms of the geometry of $\Omega$, one can then also derive geometric estimates for higher eigenvalues from such universal bounds, but this is not explored in the present paper.

Let us now put our results into the context of those known for $l=1$. Payne, Pólya and Weinberger proved in PPW1 and PPW2 that

$$
\frac{\lambda_{2}}{\lambda_{1}} \leq 3 \quad \text { for } \quad \Omega \subset \mathbb{R}^{2}
$$

and conjectured that

$$
\frac{\lambda_{2}}{\lambda_{1}} \leq\left.\frac{\lambda_{2}}{\lambda_{1}}\right|_{\text {disk }}
$$

with equality if and only if $\Omega$ is a disk. For $n \geq 2$, the analogous statements are

$$
\frac{\lambda_{2}}{\lambda_{1}} \leq 1+\frac{4}{n} \quad \text { for } \quad \Omega \subset \mathbb{R}^{n}
$$

and the $P P W$ conjecture

$$
\frac{\lambda_{2}}{\lambda_{1}} \leq\left.\frac{\lambda_{2}}{\lambda_{1}}\right|_{n-\text { ball }},
$$

with equality if and only if $\Omega$ is an $n$-ball. This important $P P W$ conjecture was solved by Ashbaugh and Benguria in AB1, AB2, AB3]. In PPW2, Payne, 
Pólya and Weinberger also proved the bound

$$
\lambda_{k+1}-\lambda_{k} \leq \frac{2}{k} \sum_{i=1}^{k} \lambda_{i}, \quad k=1,2, \cdots,
$$

for $\Omega \subset \mathbb{R}^{2}$. This result easily extends to $\Omega \subset \mathbb{R}^{n}$ as

$$
\lambda_{k+1}-\lambda_{k} \leq \frac{4}{k n} \sum_{i=1}^{k} \lambda_{i}, \quad k=1,2, \cdots .
$$

Much interesting work has been done in generalizing (1.3), e.g., in [A1], $\mathrm{A} 2$, $\mathrm{AH}$, CY1, [Ha, [HM1, [HM2, [HP, [HS, [HY], LeP, $\mathrm{Y}]$. Here we mention two results in this direction. In 1980, Hile and Protter proved [HP]

$$
\sum_{i=1}^{k} \frac{\lambda_{i}}{\lambda_{k+1}-\lambda_{i}} \geq \frac{k n}{4}, \quad \text { for } k=1,2, \cdots
$$

In 1991, Yang [Y] proved the following much stronger inequality:

$$
\sum_{i=1}^{k}\left(\lambda_{k+1}-\lambda_{i}\right)\left(\lambda_{k+1}-\left(1+\frac{4}{n}\right) \lambda_{i}\right) \leq 0, \quad \text { for } k=1,2, \cdots
$$

The inequality (1.5), as observed by Yang himself, and as later proved, e.g., in A1, [A2, $[\mathrm{AH}$, is the strongest of the classical inequalities that are derived following the scheme devised by Payne-Pólya-Weinberger. Yang's inequality provided a marked improvement for eigenvalues of large index. Recently, some Yang type inequalities on eigenvalues of the problem (1.1) for the case $l>1$ have been proved in CY2], CY3], WX1, [WX2] and [WC]. We remark that there is an error in the line below (3.1) of [WX1] where boundary terms are dropped from an integration by parts, without a reason that these terms should vanish.

For general $l$, the Payne-Pólya-Weinberg type inequality reads (cf. $[\mathrm{CQ},[\mathrm{H}]$ ):

$$
\lambda_{k+1} \leq \lambda_{k}+\frac{4 l(n+2 l-2)}{n^{2} k^{2}}\left(\sum_{i=1}^{k} \lambda_{i}^{1 / l}\right)\left(\sum_{i=1}^{k} \lambda_{i}^{(l-1) / l}\right) .
$$

In this paper, we obtain a universal inequality of Yang type for the eigenvalues of the problem (1.1) for any $l$. Indeed, we consider the more general eigenvalue problem:

$$
\begin{aligned}
& (-\Delta)^{l} u=\lambda u \text { in } M, \\
& \left.u\right|_{\partial M}=\left.\frac{\partial u}{\partial \nu}\right|_{\partial M}=\cdots=\left.\frac{\partial^{l-1} u}{\partial \nu^{l-1}}\right|_{\partial M}=0,
\end{aligned}
$$

where $M$ is a compact Riemannian manifold with boundary (possibly empty), $\Delta$ is the Laplacian operator on $M$ (for general results for the case $l=1$, see e.g. Ch]). We will prove a general inequality for the eigenvalues of the problem (1.7) (see Theorem 2.1). By using this inequality, we show that when $M$ is a bounded connected domain in $\mathbb{R}^{n}$ with smooth boundary, then the eigenvalues of the problem 
(1.1) satisfy (see Theorem 3.1):

$(1.8)$

$$
\begin{aligned}
& \sum_{i=1}^{k}\left(\lambda_{k+1}-\lambda_{i}\right)^{2} \\
& \leq\left(\frac{4 l(n+2 l-2)}{n^{2}}\right)^{1 / 2}\left(\sum_{i=1}^{k}\left(\lambda_{k+1}-\lambda_{i}\right)^{2} \lambda_{i}^{(l-1) / l}\right)^{1 / 2}\left(\sum_{i=1}^{k}\left(\lambda_{k+1}-\lambda_{i}\right) \lambda_{i}^{1 / l}\right)^{1 / 2}
\end{aligned}
$$

When $l=1,(1.8)$ is just Yang's inequality (1.5). As a consequence of (1.8), we have the following two estimates for the $(k+1)$-th eigenvalue in terms of the first $k$-eigenvalues of the problem (1.1):

$$
\begin{aligned}
& \lambda_{k+1} \\
& \leq\left\{\left(\frac{2 l(n+2 l-2)}{k^{2} n^{2}}\right)^{2}\left(\sum_{i=1}^{k} \lambda_{i}^{(l-1) / l}\right)^{2}\left(\sum_{i=1}^{k} \lambda_{i}^{1 / l}\right)^{2}-\frac{1}{k} \sum_{i=1}^{k}\left(\lambda_{i}-\frac{1}{k} \sum_{j=1}^{k} \lambda_{j}\right)^{2}\right\}^{1 / 2} \\
& +\frac{1}{k} \sum_{i=1}^{k} \lambda_{i}+\frac{2 l(n+2 l-2)}{k^{2} n^{2}}\left(\sum_{i=1}^{k} \lambda_{i}^{(l-1) / l}\right)\left(\sum_{i=1}^{k} \lambda_{i}^{1 / l}\right)
\end{aligned}
$$

$\lambda_{k+1}$

$$
\begin{aligned}
& \leq\left\{\left(\frac{2 l(n+2 l-2)}{n^{2}} \frac{1}{k} \sum_{i=1}^{k} \lambda_{i}\right)^{2}-\left(1+\frac{4 l(n+2 l-2)}{n^{2}}\right) \frac{1}{k} \sum_{j=1}^{k}\left(\lambda_{j}-\frac{1}{k} \sum_{i=1}^{k} \lambda_{i}\right)^{2}\right\}^{1 / 2} \\
& +\left(1+\frac{2 l(n+2 l-2)}{n^{2}}\right) \frac{1}{k} \sum_{i=1}^{k} \lambda_{i} .
\end{aligned}
$$

Notice that (1.9) is much stronger than (1.6).

In AB4, Ashbaugh and Benguria showed that when $l=1$, the first $n+1$ eigenvalues of the problem (1.1) satisfy the inequality

$$
\lambda_{2}+\lambda_{3}+\cdots+\lambda_{n+1} \leq(n+4) \lambda_{1} .
$$

Ashbaugh showed in A1 that when $l=2$,

$$
\lambda_{2}+\lambda_{3}+\cdots+\lambda_{n+1} \leq(n+24) \lambda_{1} .
$$

In this paper, we prove a similar inequality for any $l$ which covers the inequality (1.11) when $l=1$ and improves (1.12) when $l=2$ (cf. Theorem 4.1). The reason why the dimension $n$ comes in here is that coordinate functions in $n$-dimensional Euclidean space when used as test functions yield useful inequalities.

Consider now the so-called buckling problem:

$$
\Delta^{2} u=-\Lambda \Delta u \quad \text { in } \Omega,\left.\quad u\right|_{\partial \Omega}=\left.\frac{\partial u}{\partial \nu}\right|_{\partial \Omega}=0,
$$

where $\Omega$ is a bounded connected domain in $\mathbb{R}^{n}$. 
Let

$$
0<\Lambda_{1} \leq \Lambda_{2} \leq \Lambda_{3} \leq \cdots
$$

denote the successive eigenvalues for (1.13). Payne, Pólya and Weinberger PPW2] proved

$$
\Lambda_{2} / \Lambda_{1}<3 \quad \text { for } \Omega \subset \mathbb{R}^{2} .
$$

For $\Omega \subset \mathbb{R}^{n}$ this reads

$$
\Lambda_{2} / \Lambda_{1}<1+4 / n \text {. }
$$

Subsequently, Hile and Yeh $[\mathrm{HY}]$ obtained the improved bound

$$
\frac{\Lambda_{2}}{\Lambda_{1}} \leq \frac{n^{2}+8 n+20}{(n+2)^{2}} \quad \text { for } \Omega \subset \mathbb{R}^{n} .
$$

Ashbaugh [1] proved:

$$
\sum_{i=1}^{n} \Lambda_{i+1} \leq(n+4) \Lambda_{1}
$$

Cheng and Yang [CY2] obtained:

$$
\sum_{i=1}^{k}\left(\Lambda_{k+1}-\Lambda_{i}\right)^{2} \leq \frac{4(n+2)}{n^{2}} \sum_{i=1}^{k}\left(\Lambda_{k+1}-\Lambda_{i}\right) \Lambda_{i} .
$$

In this paper, we will prove the following inequality which strengthens (1.14) (cf. Theorem 4.2):

$$
\sum_{i=1}^{n} \Lambda_{i+1}+\frac{4\left(\Lambda_{2}-\Lambda_{1}\right)}{n+4} \leq(n+4) \Lambda_{1} .
$$

We will also show that the first $n+1$ eigenvalues of the following more general problem,

$$
\begin{aligned}
& (-\Delta)^{l} u=-\Lambda \Delta u \text { in } \Omega \\
& \left.u\right|_{\partial \Omega}=\left.\frac{\partial u}{\partial \nu}\right|_{\partial \Omega}=\cdots=\left.\frac{\partial^{l-1} u}{\partial \nu^{l-1}}\right|_{\partial \Omega}=0
\end{aligned}
$$

where $l \geq 2$ is a fixed integer, satisfy (cf. Theorem 4.3):

$$
\sum_{k=1}^{n} \frac{k}{2 l+k}\left(\Lambda_{n+2-k}-\Lambda_{1}\right)<4(l-1) \Lambda_{1} .
$$

In the final part of this paper, we will prove universal inequalities for eigenvalues of the polyharmonic operator of any order on compact domains with boundary in a unit sphere. For similar inequalities for eigenvalues of the Laplacian on compact domains in a sphere, we refer to [CY1, $[\mathrm{AH}]$ and the references therein.

\section{General inequalities for eigenvalues of the harmonic operator OF ANY ORDER ON RIEMANNIAN MANIFOLDS}

In this section, we prove some general inequalities for eigenvalues of the polyharmonic operators on compact Riemannian manifolds. 
Theorem 2.1. Let $(M,\langle\rangle$,$) be an n-dimensional compact connected Riemannian$ manifold with boundary $\partial M$ (possibly empty) and let $\nu$ be the outward unit normal vector field of $\partial M$. Let $l$ be a positive integer and denote by $\Delta$ the Laplacian operator of M. Consider the eigenvalue problem

$$
\begin{aligned}
& (-\Delta)^{l} u=\lambda u \quad \text { in } M, \\
& \left.u\right|_{\partial M}=\left.\frac{\partial u}{\partial \nu}\right|_{\partial M}=\cdots=\left.\frac{\partial^{l-1} u}{\partial \nu^{l-1}}\right|_{\partial M}=0 .
\end{aligned}
$$

Let $\lambda_{i}, i=1, \cdots$, be the $i$-th eigenvalue of the problem (2.1) and $u_{i}$ be the orthonormal eigenfunction corresponding to $\lambda_{i}$, that is,

$$
\begin{aligned}
& (-\Delta)^{l} u_{i}=\lambda_{i} u_{i} \text { in } M, \\
& \left.u_{i}\right|_{\partial M}=\left.\frac{\partial u_{i}}{\partial \nu}\right|_{\partial M}=\cdots=\left.\frac{\partial^{l-1} u_{i}}{\partial \nu^{l-1}}\right|_{\partial M}=0, \\
& \int_{M} u_{i} u_{j}=\delta_{i j}, \text { for any } i, j=1,2, \cdots .
\end{aligned}
$$

Then for any function $h \in C^{l+2}(M) \cap C^{l+1}(\partial M)$ and any positive integer $k$, we have

$$
\begin{gathered}
\sum_{i=1}^{k}\left(\lambda_{k+1}-\lambda_{i}\right)^{2} \int_{M} h u_{i}\left((-\Delta)^{l}\left(h u_{i}\right)-\lambda_{i} h u_{i}\right) \\
\leq \sum_{i=1}^{k}\left(\lambda_{k+1}-\lambda_{i}\right)\left\|\left((-\Delta)^{l}\left(h u_{i}\right)-\lambda_{i} h u_{i}\right)\right\|^{2}, \\
\sum_{i=1}^{k}\left(\lambda_{k+1}-\lambda_{i}\right)^{2} \int_{M}\left(-h u_{i}^{2} \Delta h-2 h u_{i}\left\langle\nabla h, \nabla u_{i}\right\rangle\right) \\
\left.\leq \delta \sum_{i=1}^{k}\left(\lambda_{k+1}-\lambda_{i}\right)^{2} \int_{M} h u_{i}\left((-\Delta)^{l}\left(h u_{i}\right)-\lambda_{i} h u_{i}\right)\right) \\
\quad+\sum_{i=1}^{k} \frac{\left(\lambda_{k+1}-\lambda_{i}\right)}{\delta}\left\|\left\langle\nabla h, \nabla u_{i}\right\rangle+\frac{u_{i} \Delta h}{2}\right\|^{2}
\end{gathered}
$$

and

$$
\begin{aligned}
& \sum_{i=1}^{k}\left(\lambda_{k+1}-\lambda_{i}\right)^{2} \int_{M}\left(-h u_{i}^{2} \Delta h-2 h u_{i}\left\langle\nabla h, \nabla u_{i}\right\rangle\right) \\
& \left.\leq \delta \sum_{i=1}^{k}\left(\lambda_{k+1}-\lambda_{i}\right) \|\left((-\Delta)^{l}\left(h u_{i}\right)-\lambda_{i} h u_{i}\right)\right) \|^{2} \\
& +\frac{1}{\delta} \sum_{i=1}^{k}\left(\lambda_{k+1}-\lambda_{i}\right)\left\|\left\langle\nabla h, \nabla u_{i}\right\rangle+\frac{u_{i} \Delta h}{2}\right\|^{2},
\end{aligned}
$$

where $\delta$ is any positive constant, $\|g\|^{2}=\int_{M} g^{2}$.

Proof of Theorem 2.1. The inequality (2.2) follows from Theorem 2.1 in [AH]. In fact, by taking $N=1, B_{1}=h I d, A=(-\Delta)^{l}$ in Theorem 2.1 of $[\mathrm{AH}$, one easily 
gets that

$$
\rho_{i} \equiv\left\langle\left[A, B_{1}\right] u_{i}, B_{1} u_{i}\right\rangle=\int_{M} h u_{i}\left((-\Delta)^{l}\left(h u_{i}\right)-\lambda_{i} h u_{i}\right)
$$

and

$$
\Lambda_{i} \equiv\left\|\left[A, B_{1}\right] u_{i}\right\|^{2}=\left\|\left((-\Delta)^{l}\left(h u_{i}\right)-\lambda_{i} h u_{i}\right)\right\|^{2},
$$

which, by using (2.5) in $[\mathrm{AH}$, gives (2.2).

We use some similar calculations as in CY4 to prove (2.3). For $i=1, \cdots, k$, consider the functions $\phi_{i}: M \rightarrow \mathbb{R}$ given by

$$
\phi_{i}=h u_{i}-\sum_{j=1}^{k} r_{i j} u_{j},
$$

where

$$
r_{i j}=\int_{M} h u_{i} u_{j}
$$

Since

$$
\left.\phi_{i}\right|_{\partial M}=\left.\frac{\partial \phi_{i}}{\partial \nu}\right|_{\partial M}=\cdots=\left.\frac{\partial^{l-1} \phi_{i}}{\partial \nu^{l-1}}\right|_{\partial M}=0
$$

and

$$
\int_{M} u_{j} \phi_{i}=0, \quad \forall i, j=1, \cdots, k,
$$

it follows from the Rayleigh-Ritz inequality that

$$
\begin{aligned}
\lambda_{k+1} \int_{M} \phi_{i}^{2} & \leq \int_{M} \phi_{i}(-\Delta)^{l} \phi_{i} \\
& =\lambda_{i}\left\|\phi_{i}\right\|^{2}+\int_{M} \phi_{i}\left((-\Delta)^{l} \phi_{i}-\lambda_{i} h u_{i}\right) \\
& =\lambda_{i}\left\|\phi_{i}\right\|^{2}+\int_{M} \phi_{i}\left((-\Delta)^{l}\left(h u_{i}\right)-\lambda_{i} h u_{i}\right) \\
& =\lambda_{i}\left\|\phi_{i}\right\|^{2}+\int_{M} h u_{i}\left((-\Delta)^{l}\left(h u_{i}\right)-\lambda_{i} h u_{i}\right)-\sum_{j=1}^{k} r_{i j} s_{i j}
\end{aligned}
$$

where

$$
s_{i j}=\int_{M}\left((-\Delta)^{l}\left(h u_{i}\right)-\lambda_{i} h u_{i}\right) u_{j} .
$$

Notice that if $u \in C^{l+2}(M) \cap C^{l+1}(\partial M)$ satisfies

$$
\left.u\right|_{\partial M}=\left.\frac{\partial u}{\partial \nu}\right|_{\partial M}=\cdots=\left.\frac{\partial^{l-1} u}{\partial \nu^{l-1}}\right|_{\partial M}=0,
$$

then

$$
\begin{aligned}
\left.u\right|_{\partial M} & =\left.\nabla u\right|_{\partial M}=\left.\Delta u\right|_{\partial M}=\left.\nabla(\Delta u)\right|_{\partial M}=\cdots=\left.\Delta^{m-1} u\right|_{\partial M} \\
& =\left.\nabla\left(\Delta^{m-1} u\right)\right|_{\partial M}=0, \quad \text { when } \quad l=2 m
\end{aligned}
$$


and

$$
\begin{aligned}
\left.u\right|_{\partial M} & =\left.\nabla u\right|_{\partial M}=\left.\Delta u\right|_{\partial M}=\left.\nabla(\Delta u)\right|_{\partial M}=\cdots=\left.\Delta^{m-1} u\right|_{\partial M}=\left.\nabla\left(\Delta^{m-1} u\right)\right|_{\partial M} \\
& =\left.\Delta^{m} u\right|_{\partial M}=0, \quad \text { when } \quad l=2 m+1 .
\end{aligned}
$$

Observe that both $u_{j}$ and $h u_{i}$ satisfy the boundary condition (2.8) and so they satisfy (2.9) when $l=2 m$ and (2.10) when $l=2 m+1$. Thus we can use integration by parts to conclude that

$$
\int_{M} u_{j}(-\Delta)^{l}\left(h u_{i}\right)=\int_{M} h u_{i}(-\Delta)^{l}\left(u_{j}\right)=\lambda_{j} r_{i j}
$$

which gives

$$
s_{i j}=\left(\lambda_{j}-\lambda_{i}\right) r_{i j} .
$$

Set

$$
p_{i}(h)=(-\Delta)^{l}\left(h u_{i}\right)-\lambda_{i} h u_{i}
$$

then we have from (2.7) and (2.11) that

$$
\left(\lambda_{k+1}-\lambda_{i}\right)\left\|\phi_{i}\right\|^{2} \leq \int_{M} \phi_{i} p_{i}(h)=\int_{M} h u_{i} p_{i}(h)+\sum_{j=1}^{k}\left(\lambda_{i}-\lambda_{j}\right) r_{i j}^{2} .
$$

Set

$$
t_{i j}=\int_{M} u_{j}\left(\left\langle\nabla h, \nabla u_{i}\right\rangle+\frac{u_{i} \Delta h}{2}\right)
$$

then $t_{i j}+t_{j i}=0$ and

$$
\int_{M}(-2) \phi_{i}\left(\left\langle\nabla h, \nabla u_{i}\right\rangle+\frac{u_{i} \Delta h}{2}\right)=w_{i}+2 \sum_{j=1}^{k} r_{i j} t_{i j}
$$

where

$$
w_{i}=\int_{M}\left(-h u_{i}^{2} \Delta h-2 h u_{i}\left\langle\nabla h, \nabla u_{i}\right\rangle\right)
$$


Multiplying (2.14) by $\left(\lambda_{k+1}-\lambda_{i}\right)^{2}$ and using the Schwarz inequality and (2.12), we get

$$
\begin{aligned}
& \left(\lambda_{k+1}-\lambda_{i}\right)^{2}\left(w_{i}+2 \sum_{j=1}^{k} r_{i j} t_{i j}\right) \\
= & \left(\lambda_{k+1}-\lambda_{i}\right)^{2} \int_{M}(-2) \phi_{i}\left(\left(\left\langle\nabla h, \nabla u_{i}\right\rangle+\frac{u_{i} \Delta h}{2}\right)-\sum_{j=1}^{k} t_{i j} u_{j}\right) \\
\leq & \delta\left(\lambda_{k+1}-\lambda_{i}\right)^{3}\left\|\phi_{i}\right\|^{2}+\frac{\left(\lambda_{k+1}-\lambda_{i}\right)}{\delta} \int_{M}\left|\left\langle\nabla h, \nabla u_{i}\right\rangle+\frac{u_{i} \Delta h}{2}-\sum_{j=1}^{k} t_{i j} u_{j}\right|^{2} \\
= & \delta\left(\lambda_{k+1}-\lambda_{i}\right)^{3}\left\|\phi_{i}\right\|^{2}+\frac{\left(\lambda_{k+1}-\lambda_{i}\right)}{\delta}\left(\left\|\left\langle\nabla h, \nabla u_{i}\right\rangle+\frac{u_{i} \Delta h}{2}\right\|^{2}-\sum_{j=1}^{k} t_{i j}^{2}\right) \\
\leq & \delta\left(\lambda_{k+1}-\lambda_{i}\right)^{2}\left(\int_{M} h u_{i} p_{i}(h)+\sum_{j=1}^{k}\left(\lambda_{i}-\lambda_{j}\right) r_{i j}^{2}\right) \\
& +\frac{\left(\lambda_{k+1}-\lambda_{i}\right)}{\delta}\left(\left\|\left\langle\nabla h, \nabla u_{i}\right\rangle+\frac{u_{i} \Delta h}{2}\right\|^{2}-\sum_{j=1}^{k} t_{i j}^{2}\right) .
\end{aligned}
$$

Summing over $i$ and noticing that $r_{i j}=r_{j i}, t_{i j}=-t_{j i}$, we infer

$$
\begin{aligned}
& \sum_{i=1}^{k}\left(\lambda_{k+1}-\lambda_{i}\right)^{2} w_{i}-2 \sum_{i, j=1}^{k}\left(\lambda_{k+1}-\lambda_{i}\right)\left(\lambda_{i}-\lambda_{j}\right) r_{i j} t_{i j} \\
\leq & \delta \sum_{i=1}^{k}\left(\lambda_{k+1}-\lambda_{i}\right)^{2} \int_{M} h u_{i} p_{i}(h)+\sum_{i=1}^{k} \frac{\left(\lambda_{k+1}-\lambda_{i}\right)}{\delta}\left\|\left\langle\nabla h, \nabla u_{i}\right\rangle+\frac{u_{i} \Delta h}{2}\right\|^{2} \\
& -\sum_{i, j=1}^{k}\left(\lambda_{k+1}-\lambda_{i}\right) \delta\left(\lambda_{i}-\lambda_{j}\right)^{2} r_{i j}^{2}-\sum_{i, j=1}^{k} \frac{\left(\lambda_{k+1}-\lambda_{i}\right)}{\delta} t_{i j}^{2} .
\end{aligned}
$$

Hence (2.3) is true. Substituting (2.2) into (2.3), one gets (2.4).

We end this section by listing some lemmas which are needed in the next sections.

Lemma 2.1. Let $u_{i}$ and $\lambda_{i}, i=1,2, \cdots$, be as in Theorem 2.1. Then

$$
0 \leq \int_{M} u_{i}(-\Delta)^{k} u_{i} \leq \lambda_{i}^{k / l}, \quad k=1, \cdots, l-1 .
$$

Proof. When $k \in\{1, \cdots, l-1\}$ is even, we have

$$
\int_{M} u_{i}(-\Delta)^{k} u_{i}=\int_{M} u_{i} \Delta^{k} u_{i}=\int_{M}\left(\Delta^{k / 2} u_{i}\right)^{2} \geq 0
$$


On the other hand, if $k \in\{1, \cdots, l-1\}$ is odd,

$$
\begin{aligned}
\int_{M} u_{i}(-\Delta)^{k} u_{i} & =-\int_{M} u_{i} \Delta^{k} u_{i} \\
& =-\int_{M} \Delta^{(k-1) / 2} u_{i} \Delta\left(\Delta^{(k-1) / 2} u_{i}\right) \\
& =\int_{M}\left|\nabla\left(\Delta^{(k-1) / 2} u_{i}\right)\right|^{2} \\
& \geq 0
\end{aligned}
$$

Thus the inequality at the left hand side of (2.17) holds.

We claim that for any $k=1, \cdots, l-1$,

$$
\left(\int_{M} u_{i}(-\Delta)^{k} u_{i}\right)^{k+1} \leq\left(\int_{M} u_{i}(-\Delta)^{k+1} u_{i}\right)^{k} .
$$

Since

$$
\left(\int_{M} u_{i} \Delta u_{i}\right)^{2} \leq \int_{M} u_{i}^{2} \int_{M}\left(\Delta u_{i}\right)^{2}=\int_{M} u_{i} \Delta^{2} u_{i}
$$

we know that (2.18) holds when $k=1$.

Suppose that (2.18) holds for $k-1$, that is,

$$
\left(\int_{M} u_{i}(-\Delta)^{k-1} u_{i}\right)^{k} \leq\left(\int_{M} u_{i}(-\Delta)^{k} u_{i}\right)^{k-1} \text {. }
$$

When $k$ is even, we have

$$
\begin{aligned}
\int_{M} u_{i}(-\Delta)^{k} u_{i} & =\int_{M} \Delta^{k / 2-1} u_{i} \Delta\left(\Delta^{k / 2} u_{i}\right) \\
& =-\int_{M}\left\langle\nabla\left(\Delta^{k / 2-1} u_{i}\right), \nabla\left(\Delta^{k / 2} u_{i}\right)\right\rangle \\
& \leq\left(\int_{M}\left|\nabla\left(\Delta^{k / 2-1} u_{i}\right)\right|^{2}\right)^{1 / 2}\left(\int_{M}\left|\nabla\left(\Delta^{k / 2} u_{i}\right)\right|^{2}\right)^{1 / 2} \\
& =\left(-\int_{M} \Delta^{k / 2-1} u_{i} \Delta^{k / 2} u_{i}\right)^{1 / 2}\left(-\int_{M} \Delta^{k / 2} u_{i} \Delta^{k / 2+1} u_{i}\right)^{1 / 2} \\
& =\left(\int_{M} u_{i}(-\Delta)^{k-1} u_{i}\right)^{1 / 2}\left(\int_{M} u_{i}(-\Delta)^{k+1} u_{i}\right)^{1 / 2} .
\end{aligned}
$$

On the other hand, when $k$ is odd,

$$
\begin{aligned}
\int_{M} u_{i}(-\Delta)^{k} u_{i} & =\int_{M}(-\Delta)^{(k-1) / 2} u_{i}(-\Delta)^{(k+1) / 2} u_{i} \\
& \leq\left(\int_{M}\left((-\Delta)^{(k-1) / 2} u_{i}\right)^{2}\right)^{1 / 2}\left(\int_{M}\left((-\Delta)^{(k+1) / 2} u_{i}\right)^{2}\right)^{1 / 2} \\
& =\left(\int_{M} u_{i}(-\Delta)^{k-1} u_{i}\right)^{1 / 2}\left(\int_{M} u_{i}(-\Delta)^{k+1} u_{i}\right)^{1 / 2}
\end{aligned}
$$


Thus we always have

$$
\int_{M} u_{i}(-\Delta)^{k} u_{i} \leq\left(\int_{M} u_{i}(-\Delta)^{k-1} u_{i}\right)^{1 / 2}\left(\int_{M} u_{i}(-\Delta)^{k+1} u_{i}\right)^{1 / 2} .
$$

Substituting (2.19) into (2.22), we know that (2.18) is true for $k$. Using (2.18) repeatedly, we get

$$
\int_{M} u_{i}(-\Delta)^{k} u_{i} \leq\left(\int_{M} u_{i}(-\Delta)^{k+1} u_{i}\right)^{k /(k+1)} \leq \cdots \leq\left(\int_{M} u_{i}(-\Delta)^{l} u_{i}\right)^{k / l}=\lambda_{i}^{k / l} .
$$

This shows that the inequality at the right hand side of (2.17) also holds.

Lemma 2.2. Let

$$
C=\left\{z=\left(z_{1}, \cdots, z_{n}\right) \in \mathbb{R}^{n} \mid z_{i} \geq 0, i=1, \cdots, n, \sum_{j=1}^{n} z_{j}=1\right\} .
$$

Consider the function $f: C \rightarrow R$ defined by

$$
f\left(\left(z_{1}, \cdots, z_{n}\right)\right)=\sum_{i=1}^{n} \frac{z_{i}^{2}}{1+4 z_{i}} .
$$

Then

$$
\min _{z \in C} f(z)=f\left(\left(\frac{1}{n}, \cdots, \frac{1}{n}\right)\right)=\frac{1}{n+4} .
$$

Proof of Lemma 2.2. We minimize the function

$$
\sum_{i=1}^{n} \frac{z_{i}^{2}}{1+4 z_{i}}
$$

with the constraint

$$
\sum_{j=1}^{n} z_{j}=1, z_{i} \geq 0, i=1, \cdots, n .
$$

By means of the method of the Lagrange multiplier, we consider the following function:

$$
g=\sum_{i=1}^{n} \frac{z_{i}^{2}}{1+4 z_{i}}+\lambda\left(\sum_{j=1}^{n} z_{j}-1\right),
$$

where $\lambda$ is the Lagrange multiplier. The minimum point of $\sum_{i=1}^{n} z_{i}^{2} /\left(1+4 z_{i}\right)$ is a critical point of $g$. Taking the derivative of $g$ with respect to $z_{i}$, we have

$$
\frac{2 z_{i}\left(1+4 z_{i}\right)-4 z_{i}^{2}}{\left(1+4 z_{i}\right)^{2}}+\lambda=0
$$

Multiplying the above equaltion by $\left(1+4 z_{i}\right)^{2}$ and simplifying, we get

$$
(16 \lambda+4) z_{i}^{2}+(8 \lambda+2) z_{i}+\lambda=0 .
$$


Hence at most two of the $z_{i}$ 's are distinct from each other at a critical point of g. Assume without loss of generality that $z_{1}=z_{2}=\cdots=z_{p}=s, z_{p+1}=\cdots=$ $z_{p+q}=t$ with $p+q=n$. Then $p s+q t=1$ and so we have

$$
\begin{aligned}
\sum_{i=1}^{n} \frac{z_{i}^{2}}{\left(1+4 z_{i}\right)} & =\frac{p s^{2}}{1+4 s}+\frac{q t^{2}}{1+4 t} \\
& =\frac{p s^{2}+q t^{2}+4 s t(p s+q t)}{(1+4 s)(1+4 t)} \\
& =\frac{p s^{2}+q t^{2}+4 s t}{(1+4 s)(1+4 t)} \\
& =\frac{(p+q+4)\left(p s^{2}+q t^{2}+4 s t(p s+q t)\right)}{(n+4)(1+4 s)(1+4 t)} \\
& =\frac{p^{2} s^{2}+q^{2} t^{2}+p q\left(s^{2}+t^{2}\right)+4 p s^{2}+4 q t^{2}+4(p+q) s t+16 s t}{(n+4)(1+4 s)(1+4 t)} \\
& =\frac{1-2 p q s t+p q\left(s^{2}+t^{2}\right)+4 p s^{2}+4 q t^{2}+4(p+q) s t+16 s t}{(n+4)(1+4 s)(1+4 t)} \\
& \geq \frac{1+4 p s^{2}+4 q t^{2}+4(p+q) s t+16 s t}{(n+4)(1+4 s)(1+4 t)} \\
& =\frac{1+4(p s+q t)(s+t)+16 s t}{(n+4)(1+4 s)(1+4 t)}=\frac{1}{n+4} .
\end{aligned}
$$

This completes the proof of Lemma 2.2.

Lemma 2.3. Let $\left\{a_{i}\right\}_{i=1}^{m},\left\{b_{i}\right\}_{i=1}^{m}$ and $\left\{c_{i}\right\}_{i=1}^{m}$ be three sequences of nonnegative real numbers with $\left\{a_{i}\right\}$ decreasing and $\left\{b_{i}\right\}$ and $\left\{c_{i}\right\}_{i=1}^{m}$ increasing. Then the following inequality holds:

$$
\left(\sum_{i=1}^{m} a_{i}^{2} b_{i}\right)\left(\sum_{i=1}^{m} a_{i} c_{i}\right) \leq\left(\sum_{i=1}^{m} a_{i}^{2}\right)\left(\sum_{i=1}^{m} a_{i} b_{i} c_{i}\right) .
$$

Proof. When $m=1,(2.27)$ holds trivally. Suppose that (2.27) holds when $m=k$, that is,

$$
\left(\sum_{i=1}^{k} a_{i}^{2} b_{i}\right)\left(\sum_{i=1}^{k} a_{i} c_{i}\right) \leq\left(\sum_{i=1}^{k} a_{i}^{2}\right)\left(\sum_{i=1}^{k} a_{i} b_{i} c_{i}\right) .
$$


Then when $m=k+1$, we have from (2.28) that

$$
\begin{aligned}
\text { 29) } & \left(\sum_{i=1}^{k+1} a_{i}^{2}\right)\left(\sum_{i=1}^{k+1} a_{i} b_{i} c_{i}\right)-\left(\sum_{i=1}^{k+1} a_{i}^{2} b_{i}\right)\left(\sum_{i=1}^{k+1} a_{i} c_{i}\right) \\
= & \left(\sum_{i=1}^{k} a_{i}^{2}\right)\left(\sum_{i=1}^{k} a_{i} b_{i} c_{i}\right)-\left(\sum_{i=1}^{k} a_{i}^{2} b_{i}\right)\left(\sum_{i=1}^{k} a_{i} c_{i}\right)+a_{k+1}^{2} \sum_{i=1}^{k} a_{i} b_{i} c_{i} \\
& -a_{k+1}^{2} b_{k+1} \sum_{i=1}^{k} a_{i} c_{i}+a_{k+1} b_{k+1} c_{k+1} \sum_{i=1}^{k} a_{i}^{2}-a_{k+1} c_{k+1} \sum_{i=1}^{k} a_{i}^{2} b_{i} \\
\geq & a_{k+1}^{2} \sum_{i=1}^{k} a_{i} b_{i} c_{i}-a_{k+1}^{2} b_{k+1} \sum_{i=1}^{k} a_{i} c_{i}+a_{k+1} b_{k+1} c_{k+1} \sum_{i=1}^{k} a_{i}^{2}-a_{k+1} c_{k+1} \sum_{i=1}^{k} a_{i}^{2} b_{i} \\
= & -a_{k+1}^{2} \sum_{i=1}^{k}\left(b_{k+1}-b_{i}\right) a_{i} c_{i}+a_{k+1} c_{k+1} \sum_{i=1}^{k} a_{i}^{2}\left(b_{k+1}-b_{i}\right) \\
= & \sum_{i=1}^{k} a_{k+1} a_{i}\left(b_{k+1}-b_{i}\right)\left(c_{k+1} a_{i}-a_{k+1} c_{i}\right) \\
\geq & 0,
\end{aligned}
$$

where in the last inequality we have used the fact that

$$
a_{k+1} a_{i}\left(b_{k+1}-b_{i}\right)\left(c_{k+1} a_{i}-a_{k+1} c_{i}\right) \geq 0, \quad i=1, \cdots, k .
$$

Thus (2.27) holds for $m=k+1$. This completes the proof of Lemma 2.3.

The following result is the so-called Reverse Chebyshev Inequality (cf. [HLP]).

Lemma 2.4. Suppose $\left\{a_{i}\right\}_{i=1}^{m}$ and $\left\{b_{i}\right\}_{i=1}^{m}$ are two real sequences with $\left\{a_{i}\right\}$ increasing and $\left\{b_{i}\right\}$ decreasing. Then the following inequality holds:

$$
\sum_{i=1}^{m} a_{i} b_{i} \leq \frac{1}{m}\left(\sum_{i=1}^{m} a_{i}\right)\left(\sum_{i=1}^{m} b_{i}\right) .
$$

The following lemma can also be found in HLP].

Lemma 2.5. Let $\left\{c_{k}\right\}_{k=1}^{l}$ and $\left\{d_{k}\right\}_{k=1}^{l}$ be two increasing real sequences. Then for any permutation $\left\{i_{1}, \cdots, i_{l}\right\}$ of $\{1, \cdots, l\}$, we have

$$
\sum_{k=1}^{l} c_{k} d_{i_{k}} \geq c_{1} d_{l}+c_{2} d_{l-1}+\cdots+c_{l} d_{1} .
$$

Remark. Lemma 2.4 also admits a probabilistic interpretation. We may assume that the $a_{i}$ and $b_{i}$ are nonnegative and satisfy $\sum_{i=1}^{m} a_{i}=1=\sum_{i=1}^{m} b_{i}$ so that we can interpret them as the probabilities for observing $i$ under the laws $a$ or $b$, resp. One then needs to prove that $\sum_{i=1}^{m} a_{i} b_{i} \leq \frac{1}{m}$. When the $b_{i}$ are all the same, that is, $=\frac{1}{m}$, the inequality is obviously an equality, and when $b_{i}$ is decreasing instead of being constant, the right hand side stays the same, but the left hand side can only become smaller, because then higher weights are placed on those $i$ with smaller $a_{i}$. Thus, the inequality follows. In fact, Lemma 2.5 above admits a similar interpretation. 


\section{UNIVERSAL INEQUALITIES FOR EIGENVALUES OF THE POLYHARMONIC OPERATORS ON COMPACT DOMAINS IN $\mathbb{R}^{n}$}

In this section, we will prove universal bounds on eigenvalues of the polyharmonic operator on bounded domains in a Euclidean space by using Theorem 2.1.

Theorem 3.1. Let $\Omega$ be a connected bounded domain in an n-dimensional Euclidean space $\mathbb{R}^{n}$ and let $\Delta$ be the Laplacian of $\mathbb{R}^{n}$. Denote by $\lambda_{i}$ the $i$-th eigenvalue of the eigenvalue problem:

$$
\begin{aligned}
& (-\Delta)^{l} u=\lambda u \quad \text { in } \Omega \\
& \left.u\right|_{\partial \Omega}=\left.\frac{\partial u}{\partial \nu}\right|_{\partial \Omega}=\cdots=\left.\frac{\partial^{l-1} u}{\partial \nu^{l-1}}\right|_{\partial \Omega}=0
\end{aligned}
$$

Then we have

$$
\begin{aligned}
& \sum_{i=1}^{k}\left(\lambda_{k+1}-\lambda_{i}\right)^{2} \\
& \leq\left(\frac{4 l(n+2 l-2)}{n^{2}}\right)^{1 / 2}\left(\sum_{i=1}^{k}\left(\lambda_{k+1}-\lambda_{i}\right)^{2} \lambda_{i}^{(l-1) / l}\right)^{1 / 2}\left(\sum_{i=1}^{k}\left(\lambda_{k+1}-\lambda_{i}\right) \lambda_{i}^{1 / l}\right)^{1 / 2}
\end{aligned}
$$

Corollary 3.1. Under the same assumptions as in Theorem 3.1, we have

$$
\begin{aligned}
\lambda_{k+1} \leq & \frac{1}{k} \sum_{i=1}^{k} \lambda_{i}+\frac{2 l(n+2 l-2)}{k^{2} n^{2}}\left(\sum_{i=1}^{k} \lambda_{i}^{(l-1) / l}\right)\left(\sum_{i=1}^{k} \lambda_{i}^{1 / l}\right) \\
+ & \left\{\left(\frac{2 l(n+2 l-2)}{k^{2} n^{2}}\right)^{2}\left(\sum_{i=1}^{k} \lambda_{i}^{(l-1) / l}\right)^{2}\left(\sum_{i=1}^{k} \lambda_{i}^{1 / l}\right)^{2}\right. \\
& \left.-\frac{1}{k} \sum_{i=1}^{k}\left(\lambda_{i}-\frac{1}{k} \sum_{j=1}^{k} \lambda_{j}\right)^{2}\right\}^{\frac{1}{2}} .
\end{aligned}
$$

and

$$
\begin{aligned}
\lambda_{k+1} \leq\left(1+\frac{2 l(n+2 l-2)}{n^{2}}\right) \frac{1}{k} \sum_{i=1}^{k} \lambda_{i}+\left\{\left(\frac{2 l(n+2 l-2)}{n^{2}} \frac{1}{k} \sum_{i=1}^{k} \lambda_{i}\right)^{2}\right. \\
\left.-\left(1+\frac{4 l(n+2 l-2)}{n^{2}}\right) \frac{1}{k} \sum_{i=1}^{k}\left(\lambda_{i}-\frac{1}{k} \sum_{j=1}^{k} \lambda_{j}\right)^{2}\right\}^{1 / 2} .
\end{aligned}
$$

Proof of Theorem 3.1. Let $x_{1}, x_{2}, \cdots, x_{n}$ be the standard Euclidean coordinate functions of $\mathbb{R}^{n}$. Let $u_{i}$ be the $i$-th orthonormal eigenfunction corresponding to the eigenvalue $\lambda_{i}$ of the problem (3.1), $i=1, \cdots$; then

$$
\Delta x_{\alpha}=0, \nabla x_{\alpha}=(\underbrace{0, \cdots, 0,1}_{\alpha}, 0, \cdots, 0), \quad \alpha=1,2, \cdots, n,
$$


which implies that

$$
\begin{aligned}
(-\Delta)^{l}\left(x_{\alpha} u_{i}\right) & =x_{\alpha}(-\Delta)^{l} u_{i}+2 l(-1)^{l}\left\langle\nabla x_{\alpha}, \nabla\left(\Delta^{l-1} u_{i}\right)\right\rangle \\
& =\lambda_{i} x_{\alpha} u_{i}+2 l(-1)^{l}\left\langle\nabla x_{\alpha}, \nabla\left(\Delta^{l-1} u_{i}\right)\right\rangle .
\end{aligned}
$$

Taking $h=x_{\alpha}$ in (2.3), we infer for any $\delta>0$ that

$$
\begin{aligned}
& \sum_{i=1}^{k}\left(\lambda_{k+1}-\lambda_{i}\right)^{2} \int_{\Omega}\left(-2 x_{\alpha} u_{i}\left\langle\nabla x_{\alpha}, \nabla u_{i}\right\rangle\right) \\
\leq & \delta \sum_{i=1}^{k}\left(\lambda_{k+1}-\lambda_{i}\right)^{2} \int_{\Omega} 2 l(-1)^{l} x_{\alpha} u_{i}\left\langle\nabla x_{\alpha}, \nabla\left(\Delta^{l-1} u_{i}\right)\right\rangle \\
& +\frac{1}{\delta} \sum_{i=1}^{k}\left(\lambda_{k+1}-\lambda_{i}\right)\left\|\left\langle\nabla x_{\alpha}, \nabla u_{i}\right\rangle\right\|^{2} .
\end{aligned}
$$

Summing over $\alpha$, we have

$$
\begin{aligned}
& \sum_{i=1}^{k}\left(\lambda_{k+1}-\lambda_{i}\right)^{2} \sum_{\alpha=1}^{n} \int_{\Omega}\left(-2 x_{\alpha} u_{i}\left\langle\nabla x_{\alpha}, \nabla u_{i}\right\rangle\right) \\
\leq & 2 l \delta \sum_{i=1}^{k}\left(\lambda_{k+1}-\lambda_{i}\right)^{2} \sum_{\alpha=1}^{n} \int_{\Omega}(-1)^{l} x_{\alpha} u_{i}\left\langle\nabla x_{\alpha}, \nabla\left(\Delta^{l-1} u_{i}\right)\right\rangle \\
& +\frac{1}{\delta} \sum_{i=1}^{k}\left(\lambda_{k+1}-\lambda_{i}\right) \sum_{\alpha=1}^{n}\left\|\left\langle\nabla x_{\alpha}, \nabla u_{i}\right\rangle\right\|^{2} .
\end{aligned}
$$

Observe that

$$
\sum_{\alpha=1}^{n}\left|\nabla x_{\alpha}\right|^{2}=n, \quad \sum_{\alpha=1}^{n}\left\langle\nabla x_{\alpha}, \nabla u_{i}\right\rangle^{2}=\left|\nabla u_{i}\right|^{2} .
$$

Hence

$$
\sum_{\alpha=1}^{n} \int_{\Omega}\left(-2 x_{\alpha} u_{i}\left\langle\nabla x_{\alpha}, \nabla u_{i}\right\rangle\right)=\frac{1}{2} \sum_{\alpha=1}^{n} \int_{\Omega} u_{i}^{2} \Delta x_{\alpha}^{2}=\int_{\Omega} u_{i}^{2} \sum_{\alpha=1}^{n}\left|\nabla x_{\alpha}\right|^{2}=n .
$$

From (2.17), we infer

$$
\sum_{\alpha=1}^{n}\left\|\left\langle\nabla x_{\alpha}, \nabla u_{i}\right\rangle\right\|^{2}=\int_{\Omega}\left|\nabla u_{i}\right|^{2}=\int_{\Omega}\left(-u_{i} \Delta u_{i}\right) \leq \lambda_{i}^{1 / l} .
$$

Since

$$
\Delta^{l-1}\left(x_{\alpha} u_{i}\right)=2(l-1)\left\langle\nabla x_{\alpha}, \nabla\left(\Delta^{l-2} u_{i}\right)\right\rangle+x_{\alpha} \Delta^{l-1} u_{i},
$$

we have

$$
\begin{aligned}
\int_{\Omega} x_{\alpha} u_{i}\left\langle\nabla x_{\alpha}, \nabla\left(\Delta^{l-1} u_{i}\right)\right\rangle & =\int_{\Omega} x_{\alpha} u_{i} \Delta^{l-1}\left\langle\nabla x_{\alpha}, \nabla u_{i}\right\rangle \\
& =\int_{\Omega} \Delta^{l-1}\left(x_{\alpha} u_{i}\right)\left\langle\nabla x_{\alpha}, \nabla u_{i}\right\rangle \\
& =\int_{\Omega}\left(2(l-1)\left\langle\nabla x_{\alpha}, \nabla\left(\Delta^{l-2} u_{i}\right)\right\rangle+x_{\alpha} \Delta^{l-1} u_{i}\right)\left\langle\nabla x_{\alpha}, \nabla u_{i}\right\rangle .
\end{aligned}
$$


On the other hand,

$$
\begin{aligned}
\int_{\Omega} x_{\alpha} u_{i}\left\langle\nabla x_{\alpha}, \nabla\left(\Delta^{l-1} u_{i}\right)\right\rangle & =-\int_{\Omega} \Delta^{l-1} u_{i} \operatorname{div}\left(x_{\alpha} u_{i} \nabla x_{\alpha}\right) \\
& =-\int_{\Omega} \Delta^{l-1} u_{i}\left(\left|\nabla x_{\alpha}\right|^{2} u_{i}+x_{\alpha}\left\langle\nabla x_{\alpha}, \nabla u_{i}\right\rangle\right),
\end{aligned}
$$

where $\operatorname{div}(X)$ denotes the divergence of $X$. Combining (3.10) and (3.11), we obtain

$$
\begin{aligned}
& \int_{\Omega} x_{\alpha} u_{i}\left\langle\nabla x_{\alpha}, \nabla\left(\Delta^{l-1} u_{i}\right)\right\rangle \\
= & \int_{M}\left\{(l-1)\left\langle\nabla x_{\alpha}, \nabla\left(\Delta^{l-2} u_{i}\right)\right\rangle\left\langle\nabla x_{\alpha}, \nabla u_{i}\right\rangle-\frac{1}{2} \Delta^{l-1} u_{i}\left|\nabla x_{\alpha}\right|^{2} u_{i}\right\} .
\end{aligned}
$$

It then follows from (2.17), (3.7), (3.12) and

$$
\sum_{\alpha=1}^{n}\left\langle\nabla x_{\alpha}, \nabla\left(\Delta^{l-2} u_{i}\right)\right\rangle\left\langle\nabla x_{\alpha}, \nabla u_{i}\right\rangle=\left\langle\nabla u_{i}, \nabla\left(\Delta^{l-2} u_{i}\right)\right\rangle
$$

that

$$
\begin{aligned}
& \sum_{\alpha=1}^{n} \int_{\Omega}(-1)^{l} x_{\alpha} u_{i}\left\langle\nabla x_{\alpha}, \nabla\left(\Delta^{l-1} u_{i}\right)\right\rangle \\
= & \sum_{\alpha=1}^{n} \int_{\Omega}(-1)^{l}\left\{(l-1)\left\langle\nabla x_{\alpha}, \nabla\left(\Delta^{l-2} u_{i}\right)\right\rangle\left\langle\nabla x_{\alpha}, \nabla u_{i}\right\rangle-\frac{1}{2} \Delta^{l-1} u_{i}\left|\nabla x_{\alpha}\right|^{2} u_{i}\right\} \\
= & \int_{\Omega}(-1)^{l}\left\{(l-1)\left\langle\nabla\left(\Delta^{l-2} u_{i}\right), \nabla u_{i}\right\rangle-\frac{n}{2} u_{i} \Delta^{l-1} u_{i}\right\} \\
= & \left(l-1+\frac{n}{2}\right) \int_{\Omega} u_{i}(-\Delta)^{l-1} u_{i} \\
\leq & \left(l-1+\frac{n}{2}\right) \lambda_{i}^{(l-1) / l} .
\end{aligned}
$$

Substituting (3.8), (3.9) and (3.13) into (3.6), one gets

$$
\begin{aligned}
& n \sum_{i=1}^{k}\left(\lambda_{k+1}-\lambda_{i}\right)^{2} \\
\leq & l(n+2 l-2) \delta \sum_{i=1}^{k}\left(\lambda_{k+1}-\lambda_{i}\right)^{2} \lambda_{i}^{(l-1) / l}+\frac{1}{\delta} \sum_{i=1}^{k}\left(\lambda_{k+1}-\lambda_{i}\right) \lambda_{i}^{1 / l} .
\end{aligned}
$$

Taking

$$
\delta=\left\{\frac{\sum_{i=1}^{k}\left(\lambda_{k+1}-\lambda_{i}\right) \lambda_{i}^{1 / l}}{l(n+2 l-2) \sum_{i=1}^{k}\left(\lambda_{k+1}-\lambda_{i}\right)^{2} \lambda_{i}^{(l-1) / l}}\right\}^{1 / 2},
$$

we get (3.1).

In the proof of Corollary 3.1 we will use the reverse-Chebyshev inequality which was used earlier for similar purposes in [A1] and $\mathrm{AH}$ ].

Proof of Corollary 3.1. It follows from (2.30) that

$$
\sum_{i=1}^{k}\left(\lambda_{k+1}-\lambda_{i}\right) \lambda_{i}^{1 / l} \leq \frac{1}{k}\left(\sum_{i=1}^{k}\left(\lambda_{k+1}-\lambda_{i}\right)\right)\left(\sum_{i=1}^{k} \lambda_{i}^{1 / l}\right)
$$


and

$$
\sum_{i=1}^{k}\left(\lambda_{k+1}-\lambda_{i}\right)^{2} \lambda_{i}^{(l-1) / l} \leq \frac{1}{k}\left(\sum_{i=1}^{k}\left(\lambda_{k+1}-\lambda_{i}\right)^{2}\right)\left(\sum_{i=1}^{k} \lambda_{i}^{(l-1) / l}\right) .
$$

Introducing (3.15) and (3.16) into (3.1), we infer

$$
\begin{aligned}
& \sum_{i=1}^{k}\left(\lambda_{k+1}-\lambda_{i}\right)^{2} \\
\leq & \frac{4 l(n+2 l-2)}{k^{2} n^{2}}\left(\sum_{i=1}^{k}\left(\lambda_{k+1}-\lambda_{i}\right)\right)\left(\sum_{i=1}^{k} \lambda_{i}^{(l-1) / l}\right)\left(\sum_{i=1}^{k} \lambda_{i}^{1 / l}\right) .
\end{aligned}
$$

Solving this quadratic polynomial about $\lambda_{k+1}$, one gets (3.2).

From (2.27), we have

$$
\begin{aligned}
& \left(\sum_{i=1}^{k}\left(\lambda_{k+1}-\lambda_{i}\right)^{2} \lambda_{i}^{(l-1) / l}\right)\left(\sum_{i=1}^{k}\left(\lambda_{k+1}-\lambda_{i}\right) \lambda_{i}^{1 / l}\right) \\
\leq & \left(\sum_{i=1}^{k}\left(\lambda_{k+1}-\lambda_{i}\right)^{2}\right)\left(\sum_{i=1}^{k}\left(\lambda_{k+1}-\lambda_{i}\right) \lambda_{i}\right) .
\end{aligned}
$$

It then follows from (3.1) that

$$
\sum_{i=1}^{k}\left(\lambda_{k+1}-\lambda_{i}\right)^{2} \leq \frac{4 l(n+2 l-2)}{n^{2}} \sum_{i=1}^{k}\left(\lambda_{k+1}-\lambda_{i}\right) \lambda_{i},
$$

which implies (3.3).

\section{UNIVERSAL INEQUALITIES FOR LOWER ORDER EIGENVALUES OF THE} POLYHARMONIC OPERATORS ON COMPACT DOMAINS IN $\mathbb{R}^{n}$

In AB4, Ashbaugh and Benguria showed that when $l=1$, the first $n+1$ eigenvalues of the problem (1.1) satisfy the inequality $\lambda_{2}+\lambda_{3}+\cdots+\lambda_{n+1} \leq(n+4) \lambda_{1}$. Also, Ashbaugh showed in [A1] that when $l=2, \lambda_{2}+\lambda_{3}+\cdots+\lambda_{n+1} \leq(n+24) \lambda_{1}$. The following result generalizes the estimate by Ashbaugh and Benguria to any $l$ and strengthens the above Ashbaugh's inequality.

Theorem 4.1. Under the same assumptions as in Theorem 3.1, we have

$$
\sum_{i=2}^{n+1} \lambda_{i}+\sum_{i=1}^{n-1} \frac{2(l-1) i}{2 l+i-1}\left(\lambda_{n+1-i}-\lambda_{1}\right) \leq(n+4 l(2 l-1)) \lambda_{1} .
$$

Proof of Theorem 3.2. As in the proof of Theorem 3.1, we let $u_{i}$ be the $i$-th orthonormal eigenfunction corresponding to the eigenvalue $\lambda_{i}$ of the problem (3.1), $i=1, \cdots$. We first claim that there exists a Cartesian coordinate system $\left(x_{1}, \ldots, x_{n}\right)$ of $\mathbb{R}^{n}$ so that the following orthogonality conditions are satisfied:

$$
\int_{\Omega} x_{i} u_{1} u_{j}=0 \text { for } 1 \leq j \leq i \leq n .
$$

Indeed, by choosing the origin properly, we can assume that there exist Cartesian coordinates $\left(y_{1}, \ldots, y_{n}\right)$ of $R^{n}$ such that

$$
\int_{\Omega} y_{i} u_{1}^{2}=0 \quad \text { for } \quad i=1, \ldots, n \text {. }
$$


Consider the matrix $A$ defined by

$$
A=\left[\begin{array}{cccc}
\int_{\Omega} y_{1} u_{1} u_{2} & \int_{\Omega} y_{1} u_{1} u_{3} & \cdots & \int_{\Omega} y_{1} u_{1} u_{n+1} \\
\int_{\Omega} y_{2} u_{1} u_{2} & \int_{\Omega} y_{2} u_{1} u_{2} & \cdots & \int_{\Omega} y_{2} u_{1} u_{n+1} \\
\cdots & \cdots & \cdots & \cdots \\
\int_{\Omega} y_{n} u_{1} u_{2} & \int_{\Omega} y_{n} u_{1} u_{3} & \cdots & \int_{\Omega} y_{n} u_{1} u_{n+1}
\end{array}\right] .
$$

From the orthogonalization of Gram-Schmidt (QR-factorization theorem), we know that $A$ can be written as

$$
B=T A,
$$

where $T=\left(t_{i j}\right)$ is an orthogonal $n \times n$ matrix and $B$ is an upper triangular matrix. Hence, we have, for any $k$ and $j$ with $k>j$,

$$
\sum_{l=1}^{n} t_{k l} \int_{\Omega} y_{l} u_{1} u_{j+1}=0 .
$$

Defining new coordinate functions $x_{k}$, by $x_{k}=\sum_{j=1}^{n} t_{k j} y_{j}$, one has, for any $i, j=$ $1,2, \ldots, n$, satisfying $i>j$,

$$
\int_{\Omega} x_{i} u_{1} u_{j+1}=0
$$

Combining (4.3) and (4.4), we know that our claim is true.

Since (4.2) holds, for each $i=1, \ldots, n$, we get from the Rayleigh-Ritz inequality, (3.5) and (3.12) that

$$
\begin{aligned}
\lambda_{i+1} & \leq \frac{\int_{\Omega} x_{i} u_{1}(-\Delta)^{l}\left(x_{i} u_{1}\right)}{\int_{\Omega} x_{i}^{2} u_{1}^{2}} \\
& =\lambda_{1}+\frac{2 l \int_{\Omega}(-1)^{l} x_{i} u_{1}\left\langle\nabla x_{i}, \nabla\left(\Delta^{l-1} u_{1}\right)\right\rangle}{\int_{\Omega} x_{i}^{2} u_{1}^{2}} \\
& =\lambda_{1}+\frac{2 l \int_{\Omega}(-1)^{l}\left\{(l-1)\left\langle\nabla x_{i}, \nabla\left(\Delta^{l-2} u_{1}\right)\right\rangle\left\langle\nabla x_{i}, \nabla u_{1}\right\rangle-\frac{1}{2} u_{1} \Delta^{l-1} u_{1}\right\}}{\int_{\Omega} x_{i}^{2} u_{1}^{2}} .
\end{aligned}
$$

Since

$$
1=\int_{\Omega} u_{1}^{2}=-2 \int_{\Omega} x_{i} u_{1}\left\langle\nabla x_{i}, \nabla u_{1}\right\rangle,
$$

we have by squaring both sides and using the Cauchy-Schwarz inequality that

$$
1 \leq 4 \int_{\Omega}\left\langle\nabla x_{i}, \nabla u_{1}\right\rangle^{2} \int_{\Omega} x_{i}^{2} u_{1}^{2}
$$

which gives

$$
\frac{1}{\int_{\Omega} x_{i}^{2} u_{1}^{2}} \leq 4 \int_{\Omega}\left\langle\nabla x_{i}, \nabla u_{1}\right\rangle^{2} .
$$

Substituting (4.6) into (4.5) yields

$$
\begin{aligned}
\lambda_{i+1} & -\lambda_{1} \\
& \leq\left(2 l \int_{\Omega}(-1)^{l}\left\{(l-1)\left\langle\nabla x_{i}, \nabla\left(\Delta^{l-2} u_{1}\right)\right\rangle\left\langle\nabla x_{i}, \nabla u_{1}\right\rangle-\frac{1}{2} u_{1} \Delta^{l-1} u_{1}\right\}\right) \\
& \times\left(4 \int_{\Omega}\left\langle\nabla x_{i}, \nabla u_{1}\right\rangle^{2}\right) .
\end{aligned}
$$


Set

(4.8)

$a=\int_{\Omega} u_{1}(-\Delta)^{l-1} u_{1}, \quad a_{i}=\int_{\Omega}(-1)^{l}\left\langle\nabla x_{i}, \nabla\left(\Delta^{l-2} u_{1}\right)\right\rangle\left\langle\nabla x_{i}, \nabla u_{1}\right\rangle, \quad i=1, \cdots, n ;$

then

$$
\sum_{i=1}^{n} a_{i}=a
$$

Taking a permutation $\left\{i_{1}, \cdots, i_{n}\right\}$ of $\{1, \cdots, n\}$ so that

$$
a_{i_{n}} \leq a_{i_{n-1}} \leq \cdots \leq a_{i_{1}}
$$

it then follows from (4.9) and (4.10) that

$$
a_{i_{k}} \leq \frac{1}{k} a, k=1, \cdots, n .
$$

Substituting (4.11) into (4.7), we get

$$
\lambda_{i_{k}+1}-\lambda_{1} \leq 8 l\left(\frac{(l-1)}{k} a+\frac{a}{2}\right) \int_{\Omega}\left\langle\nabla x_{i_{k}}, \nabla u_{1}\right\rangle^{2} .
$$

Multiplying (4.12) by $\frac{(2 l-1) k}{2(l-1)+k}$ and simplifying, one has

$$
\begin{aligned}
\left(1+\frac{2(l-1)(k-1)}{2(l-1)+k}\right)\left(\lambda_{i_{k}+1}-\lambda_{1}\right) & =\frac{(2 l-1) k}{2(l-1)+k}\left(\lambda_{i_{k}+1}-\lambda_{1}\right) \\
& \leq 4 l(2 l-1) a \int_{\Omega}\left\langle\nabla x_{i_{k}}, \nabla u_{1}\right\rangle^{2} .
\end{aligned}
$$

Summing over $k$, we have

$$
\begin{gathered}
4 l(2 l-1) a \int_{\Omega}\left|\nabla u_{1}\right|^{2} \geq \sum_{k=1}^{n}\left(1+\frac{2(l-1)(k-1)}{2(l-1)+k}\right)\left(\lambda_{i_{k}+1}-\lambda_{1}\right) \\
=\sum_{k=1}^{n}\left(\lambda_{k}-\lambda_{1}\right)+\sum_{k=2}^{n} \frac{2(l-1)(k-1)}{2(l-1)+k}\left(\lambda_{i_{k}+1}-\lambda_{1}\right) .
\end{gathered}
$$

Observe that $\left\{i_{1}, \cdots, i_{n}\right\}$ is a permutation of $\{1, \cdots, n\}$. We claim that there is a permutation $\left\{q_{2}, q_{3}, \cdots, q_{n}\right\}$ of $\{1, \ldots, n-1\}$ such that

$$
\sum_{k=2}^{n} \frac{2(l-1)(k-1)}{2(l-1)+k}\left(\lambda_{i_{k}+1}-\lambda_{1}\right) \geq \sum_{k=2}^{n} \frac{2(l-1)(k-1)}{2(l-1)+k}\left(\lambda_{q_{k}+1}-\lambda_{1}\right) .
$$

In fact, if $i_{1}=n$, then $\left\{i_{2}, \cdots, i_{n}\right\}$ is a permutation of $\{1, \cdots, n-1\}$ and there is nothing to prove. On the other hand, if $i_{1}=m \in\{1,2, \cdots, n-1\}$, then $\left\{i_{2}, i_{3}, \cdots, i_{n}\right\}=\{1,2, \cdots, m-1, m+1, \cdots, n\}$ and so there is a $j \in\{2, \cdots, n\}$ such that $i_{j}=n$, which implies that

$$
\left\{i_{2}, i_{3}, \cdots, i_{j-1}, i_{j+1}, \cdots, i_{n}\right\}=\{1,2, \cdots, m-1, m+1, \cdots, n-1\}
$$


and

$$
\begin{aligned}
& \sum_{k=2}^{n} \frac{2(l-1)(k-1)}{2(l-1)+k}\left(\lambda_{i_{k}+1}-\lambda_{1}\right) \\
= & \sum_{k=2, k \neq j}^{n} \frac{2(l-1)(k-1)}{2(l-1)+k}\left(\lambda_{i_{k}+1}-\lambda_{1}\right)+\frac{2(l-1)(j-1)}{2(l-1)+j}\left(\lambda_{n+1}-\lambda_{1}\right) \\
\geq & \sum_{k=2, k \neq j}^{n} \frac{2(l-1)(k-1)}{2(l-1)+k}\left(\lambda_{i_{k}+1}-\lambda_{1}\right)+\frac{2(l-1)(j-1)}{2(l-1)+j}\left(\lambda_{m+1}-\lambda_{1}\right) .
\end{aligned}
$$

From (4.16), we know that $\left\{i_{2}, i_{3}, \cdots, i_{j-1}, m, i_{j+1}, \cdots, i_{n}\right\}$ is a permutation of $\{1,2, \cdots, m-1, m, m+1, \cdots, n-1\}$. Set $i_{2}=q_{2}, \cdots, i_{j-1}=q_{j-1}, m=q_{j}, i_{j+1}=$ $q_{j+1}, \cdots, i_{n}=q_{n}$; then $\left\{q_{2}, q_{3}, \cdots, q_{n}\right\}$ is a permutation of $\{1, \ldots, n-1\}$ and we can rewrite (4.17) as (4.15). Thus our claim is true.

Since $\left\{\frac{2(l-1)(k-1)}{2(l-1)+k}\right\}_{k=2}^{n}$ and $\left\{\lambda_{k}-\lambda_{1}\right\}_{k=2}^{n}$ are two increasing sequences and $\left\{q_{1}+\right.$ $\left.1, q_{2}+1, \cdots, q_{n}+1\right\}$ is a permutation of $\{2, \cdots, n\}$, we conclude from Lemma 2.5 that

$$
\sum_{k=2}^{n} \frac{2(l-1)(k-1)}{2(l-1)+k}\left(\lambda_{q_{k}+1}-\lambda_{1}\right) \geq \sum_{k=2}^{n} \frac{2(l-1)(k-1)}{2(l-1)+k}\left(\lambda_{n-k+2}-\lambda_{1}\right) .
$$

Thus we have from (2.17), (4.14) and (4.18) that

$$
\begin{aligned}
& \sum_{k=1}^{n} \lambda_{i+1}+\sum_{k=2}^{n} \frac{2(l-1)(k-1)}{2(l-1)+k}\left(\lambda_{n-k+2}-\lambda_{1}\right) \\
\leq & n \lambda_{1}+4 l(2 l-1) a \int_{\Omega}\left|\nabla u_{1}\right|^{2} \\
= & n \lambda_{1}+4 l(2 l-1)\left(\int_{\Omega} u_{1}(-\Delta)^{l-1} u_{1}\right)\left(\int_{\Omega} u_{1}\left(-\Delta u_{1}\right)\right) \\
\leq & n \lambda_{1}+4 l(2 l-1) \lambda_{1}^{(l-1) / l} \cdot \lambda_{1}^{1 / l}=(n+4 l(2 l-1)) \lambda_{1} .
\end{aligned}
$$

This is just the inequality (4.1). The proof of Theorem 3.2 is completed.

Our next result is to prove the inequality (1.16) as mentioned in the introduction.

Theorem 4.2. Let $\Omega$ be a connected bounded domain with smooth boundary in $\mathbb{R}^{n}$. Denote by $\nu$ the outward unit normal vector field of $\partial \Omega$ and let $\Lambda_{i}, i=1, \cdots, n+1$, be the first $n+1$ eigenvalues of the following buckling problem:

$$
\Delta^{2} u=-\Lambda \Delta u \quad \text { in } \Omega,\left.\quad u\right|_{\partial \Omega}=\left.\frac{\partial u}{\partial \nu}\right|_{\partial \Omega}=0 .
$$

Then,

$$
\sum_{i=1}^{n} \Lambda_{i+1}+\frac{4\left(\Lambda_{2}-\Lambda_{1}\right)}{n+4} \leq(n+4) \Lambda_{1} .
$$

Proof of Theorem 4.2. Let us denote by $u_{i}$ the $i$-th orthonormal eigenfunction corresponding to the eigenvalue $\lambda_{i}$ of the buckling problem (4.20), $i=1, \cdots$. That is, 
we have

$$
\begin{array}{r}
\Delta^{2} u_{i}=-\Lambda_{i} \Delta u \quad \text { in } \Omega,\left.\quad u_{i}\right|_{\partial \Omega}=\left.\frac{\partial u_{i}}{\partial \nu}\right|_{\partial \Omega}=0 \\
\int_{\Omega}\left\langle\nabla u_{i}, \nabla u_{j}\right\rangle=\delta_{i j}, \quad \forall i, j=1, \cdots .
\end{array}
$$

Using similar discussions as in the proof of Theorem 4.1, we can find a set of Cartesian coordinates $\left(x_{1}, \ldots, x_{n}\right)$ of $\mathbb{R}^{n}$ so that the following orthogonality conditions are satisfied:

$$
\int_{\Omega}\left\langle\nabla\left(x_{i} u_{1}\right), \nabla u_{j}\right\rangle=-\int_{\Omega} x_{i} u_{1} \Delta u_{j}=0 \quad \text { for } \quad 1 \leq j \leq i \leq n .
$$

Now we start with the well-known Rayleigh-Ritz inequality

$$
\Lambda_{i+1} \leq \frac{\int_{\Omega} \phi \Delta^{2} \phi}{\int_{\Omega}|\nabla \phi|^{2}}
$$

which is satisfied by any sufficiently smooth function $\phi$ such that

$$
\begin{aligned}
& \phi=\frac{\partial \phi}{\partial \nu}=0 \text { on } \partial \Omega, \\
& \int_{\Omega}\left\langle\nabla \phi, \nabla u_{j}\right\rangle=0, j=1, \cdots, i .
\end{aligned}
$$

Setting $u=u_{1}$, we choose as our trial function

$$
\phi=x_{i} u,
$$

which clearly satisfies the above boundary condition, and by (4.24) the orthogonality condition also. Thus we have

$$
\Lambda_{i+1} \int_{\Omega}\left|\nabla\left(x_{i} u\right)\right|^{2} \leq \int_{\Omega} x_{i} u \Delta^{2}\left(x_{i} u\right), \quad i=1, \cdots, n .
$$

Let us calculate

$$
\int_{\Omega}\left|\nabla\left(x_{i} u\right)\right|^{2}=\int_{\Omega} x_{i}^{2}|\nabla u|^{2}+2 \int_{\Omega} x_{i} u u_{x_{i}}+\int_{\Omega} u^{2}=\int_{\Omega} x_{i}^{2}|\nabla u|^{2},
$$

where $u_{x_{i}}=\left\langle\nabla x_{i}, \nabla u\right\rangle=\frac{\partial u}{\partial x_{i}}$. As for the right hand side of (4.26), we have

$$
\begin{aligned}
\int_{\Omega} x_{i} u \Delta^{2}\left(x_{i} u\right) & =\int_{\Omega} x_{i} u\left(x_{i} \Delta^{2} u+4 \Delta u_{x_{i}}\right) \\
& =-\Lambda_{1} \int_{\Omega} x_{i}^{2} u \Delta u+4 \int_{\Omega} x_{i} u \Delta u_{x_{i}} .
\end{aligned}
$$

By integration by parts, one gets

$$
\begin{aligned}
\int_{\Omega} x_{i}^{2} u \Delta u & =-\int_{\Omega}\left\langle\nabla\left(x_{i}^{2} u\right), \nabla u\right\rangle \\
& =-\int_{\Omega} x_{i}^{2}|\nabla u|^{2}-2 \int_{\Omega} x_{i} u u_{x_{i}} \\
& =-\int_{\Omega} x_{i}^{2}|\nabla u|^{2}+\int_{\Omega} u^{2} .
\end{aligned}
$$

Thus,

$$
\int_{\Omega} x_{i} u \Delta^{2}\left(x_{i} u\right)=\Lambda_{1} \int_{\Omega} x_{i}^{2}|\nabla u|^{2}-\Lambda_{1} \int_{\Omega} u^{2}+4 \int_{\Omega} x_{i} u \Delta u_{x_{i}} .
$$


Substituting (4.27) and (4.30) into (4.26) and dividing both sides by $\int_{\Omega} x_{i}^{2}|\nabla u|^{2}$, we get

$$
\Lambda_{i+1}-\Lambda_{1} \leq \frac{-\Lambda_{1} \int_{\Omega} u^{2}+4 \int_{\Omega} x_{i} u \Delta u_{x_{i}}}{\int_{\Omega} x_{i}^{2}|\nabla u|^{2}}
$$

We have

$$
\begin{aligned}
\int_{\Omega} x_{i} u \Delta u_{x_{i}} & =-\int_{\Omega}\left\langle\nabla\left(x_{i} u\right), \nabla u_{x_{i}}\right\rangle \\
& =-\int_{\Omega}\left(x_{i}\left\langle\nabla u, \nabla u_{x_{i}}\right\rangle+u u_{x_{i} x_{i}}\right) \\
& =\frac{1}{2} \int_{\Omega}|\nabla u|^{2}+\int_{\Omega} u_{x_{i}}^{2} \\
& =\frac{1}{2}+\int_{\Omega} u_{x_{i}}^{2} .
\end{aligned}
$$

The Cauchy-Schwarz inequality implies that

$$
\begin{aligned}
1 & =\int_{\Omega}(-u \Delta u)=\left(\int_{\Omega}(-u \Delta u)\right)^{2} \leq\left(\int_{\Omega} u^{2}\right)\left(\int_{\Omega}(\Delta u)^{2}\right) \\
& =\left(\int_{\Omega} u^{2}\right)\left(\int_{\Omega} u \Delta^{2} u\right)=\left(\int_{\Omega} u^{2}\right) \Lambda_{1} \int_{\Omega}|\nabla u|^{2}=\Lambda_{1} \int_{\Omega} u^{2} .
\end{aligned}
$$

Substituting (4.32) and (4.33) into (4.31), we get

$$
\Lambda_{i+1}-\Lambda_{1} \leq \frac{1+4 \int_{\Omega} u_{x_{i}}^{2}}{\int_{\Omega} x_{i}^{2}|\nabla u|^{2}}
$$

Since

$$
\begin{aligned}
\left(\int_{\Omega}\left\langle\nabla\left(x_{i} u\right), \nabla u_{x_{i}}\right\rangle\right)^{2} & \leq\left(\int_{\Omega}\left|\nabla\left(x_{i} u\right)\right|^{2}\right)\left(\int_{\Omega}\left|\nabla u_{x_{i}}\right|^{2}\right) \\
& =\left(\int_{\Omega} x_{i}^{2}|\nabla u|^{2}\right)\left(\int_{\Omega}\left|\nabla u_{x_{i}}\right|^{2}\right),
\end{aligned}
$$

it follows that

$$
\frac{\left(\int_{\Omega}\left\langle\nabla\left(x_{i} u\right), \nabla u_{x_{i}}\right\rangle\right)^{2}}{\int_{\Omega} x_{i}^{2}|\nabla u|^{2}} \leq \int_{\Omega}\left|\nabla u_{x_{i}}\right|^{2} .
$$

Combining (4.32) and (4.36), one gets

$$
\frac{1+4 \int_{\Omega} u_{x_{i}}^{2}+4\left(\int_{\Omega} u_{x_{i}}^{2}\right)^{2}}{\int_{\Omega} x_{i}^{2}|\nabla u|^{2}} \leq 4 \int_{\Omega}\left|\nabla u_{x_{i}}\right|^{2}
$$

Set

$$
b_{i}=\int_{\Omega} u_{x_{i}}^{2} \quad \text { and } \quad \epsilon_{i}=\frac{\left(\int_{\Omega} u_{x_{i}}^{2}\right)^{2}}{1+4 \int_{\Omega} u_{x_{i}}^{2}} .
$$

It then follows from (4.34) and (4.37) that

$$
\left(1+4 \epsilon_{i}\right)\left(\Lambda_{i+1}-\Lambda_{1}\right) \leq 4 \int_{\Omega}\left|\nabla u_{x_{i}}\right|^{2} .
$$


Since $\left.u\right|_{\partial \Omega}=\left.\frac{\partial u}{\partial \nu}\right|_{\partial \Omega}=0$, we know that $\left.u_{x_{i}}\right|_{\partial \Omega}=0$, which implies from the divergence theorem that

$$
\int_{\Omega}\left|\nabla u_{x_{i}}\right|^{2}=-\int_{\Omega} u_{x_{i}} \Delta u_{x_{i}}=-\int_{\Omega} u_{x_{i}}(\Delta u)_{x_{i}} .
$$

Let $e_{i}=(\underbrace{0, \cdots, 0,1}_{i}, 0, \cdots, 0)$ and consider the vector field $X=u(\Delta u)_{x_{i}} e_{i}$. We infer from the divergence theorem and $\left.X\right|_{\partial \Omega}=0$ that

$$
0=\int_{\Omega} \operatorname{div} X=\int_{\Omega}\left(u_{x_{i}}(\Delta u)_{x_{i}}+u(\Delta u)_{x_{i} x_{i}}\right) .
$$

Hence, we have

$$
\int_{\Omega}\left|\nabla u_{x_{i}}\right|^{2}=\int_{\Omega} u(\Delta u)_{x_{i} x_{i}}
$$

Substituting (4.40) into (4.39) and summing on $i$ from 1 to $n$, we have

$$
\begin{aligned}
\sum_{i=1}^{n}\left(1+4 \epsilon_{i}\right)\left(\Lambda_{i+1}-\Lambda_{1}\right) & \leq 4 \int_{\Omega} u\left(\sum_{i=1}^{n}(\Delta u)_{x_{i} x_{i}}\right) \\
& =-4 \Lambda_{1} \int_{\Omega} u \Delta u \\
& =4 \Lambda_{1} \int_{\Omega}|\nabla u|^{2}=4 \Lambda_{1} .
\end{aligned}
$$

Now we want to estimate the left hand side of the above inequality. We have

$$
\sum_{i=1}^{n}\left(1+4 \epsilon_{i}\right)\left(\Lambda_{i+1}-\Lambda_{1}\right) \geq \sum_{i=1}^{n}\left(\Lambda_{i+1}-\Lambda_{1}\right)+4\left(\Lambda_{2}-\Lambda_{1}\right) \sum_{i=1}^{n} \epsilon_{i} .
$$

From the definition, we know that

$$
\sum_{i=1}^{n} b_{i}=\int_{\Omega}|\nabla u|^{2}=1
$$

Thus we deduce from Lemma 2.2 that

$$
\sum_{i=1}^{n} \epsilon_{i}=f\left(\left(b_{1}, \cdots, b_{n}\right)\right) \geq \frac{1}{n+4}
$$

Combining (4.41), (4.42) and (4.44), we get

$$
\sum_{i=2}^{n+1} \Lambda_{i}+\frac{4\left(\Lambda_{2}-\Lambda_{1}\right)}{n+4} \leq(n+4) \Lambda_{1} .
$$

This completes the proof of Theorem 4.2.

The final result of this section is to prove the inequality (1.18). That is, we have

Theorem 4.3. Let $l \geq 2$ be a positive integer and let $\Omega$ be a connected bounded domain with smooth boundary in $\mathbb{R}^{n}$. Consider the eigenvalue problem

$$
\begin{aligned}
& (-\Delta)^{l} u=-\Lambda \Delta u \quad \text { in } \Omega \\
& \left.u\right|_{\partial \Omega}=\left.\frac{\partial u}{\partial \nu}\right|_{\partial \Omega}=\cdots=\left.\frac{\partial^{l-1} u}{\partial \nu^{l-1}}\right|_{\partial \Omega}=0
\end{aligned}
$$


Let

$$
0<\Lambda_{1} \leq \Lambda_{2} \leq \cdots \leq \Lambda_{n+1}
$$

denote the first $n+1$ eigenvalues of the above problem. Then we have

$$
\sum_{k=1}^{n} \frac{k}{2 l+k}\left(\Lambda_{n+2-k}-\Lambda_{1}\right)<4(l-1) \Lambda_{1} .
$$

Proof of Theorem 4.3. Let $v_{i}$ be the $i$-th orthonormal eigenfunction of the problem (4.46) corresponding to the eigenvalue $\Lambda_{i}, i=1,2, \cdots$, that is, $v_{i}$ satisfies

$$
\begin{aligned}
& (-\Delta)^{l} v_{i}=-\Lambda_{i} \Delta v_{i} \text { in } \Omega, \\
& \left.v_{i}\right|_{\partial \Omega}=\left.\frac{\partial v_{i}}{\partial \nu}\right|_{\partial \Omega}=0=\cdots=\left.\frac{\partial^{l-1} v_{i}}{\partial \nu^{l-1}}\right|_{\partial \Omega}=0, \\
& \int_{\Omega}\left\langle\nabla v_{i}, \nabla v_{j}\right\rangle=\delta_{i j}, \quad \forall i, j .
\end{aligned}
$$

As in the proof of Theorem 4.1, we take a set of Cartesian coordinates $\left(x_{1}, \ldots, x_{n}\right)$ of $\mathbb{R}^{n}$ so that the following orthogonality conditions are satisfied:

$$
\int_{\Omega}\left\langle\nabla\left(x_{i} v_{1}\right), \nabla v_{j}\right\rangle=-\int_{\Omega} x_{i} v_{1} \Delta v_{j}=0 \quad \text { for } \quad 1 \leq j \leq i \leq n .
$$

The Rayleigh-Ritz inequality now states that

$$
\Lambda_{i+1} \leq \frac{\int_{\Omega} \phi(-\Delta)^{l} \phi}{\int_{\Omega}|\nabla \phi|^{2}},
$$

which is satisfied by any sufficiently smooth function $\phi$ such that

$$
\begin{gathered}
\phi=\frac{\partial \phi}{\partial \nu}=\cdots=\frac{\partial^{l-1} \phi}{\partial \nu^{l-1}}=0 \text { on } \partial \Omega, \\
\int_{\Omega}\left\langle\nabla \phi, \nabla v_{j}\right\rangle=0, j=1, \cdots, i .
\end{gathered}
$$

Setting $v=v_{1}$, we choose as our trial function $\phi=x_{i} v$, which clearly satisfies the above boundary condition, and by (4.48) the orthogonality condition also. Then we have

$$
\Lambda_{i+1} \leq \frac{\int_{\Omega} x_{i} v(-\Delta)^{l}\left(x_{i} v\right)}{\int_{\Omega}\left|\nabla\left(x_{i} v\right)\right|^{2}}, i=1, \cdots, n .
$$

Since $\Delta x_{i}=0, i=1,2, \cdots, n$, we have

$$
\begin{aligned}
(-\Delta)^{l}\left(x_{i} v\right) & =x_{i}(-\Delta)^{l} v+2 l(-1)^{l}\left(\Delta^{l-1} v\right)_{x_{i}} \\
& =\Lambda_{1} x_{i}(-\Delta v)+2 l(-1)^{l}\left(\Delta^{l-1} v\right)_{x_{i}} .
\end{aligned}
$$

As calculated in (4.27) and (4.29), we have

$$
\int_{\Omega}\left|\nabla\left(x_{i} v\right)\right|^{2}=\int_{\Omega} x_{i}^{2}|\nabla v|^{2}, \int_{\Omega} x_{i}^{2} v \Delta v=-\int_{\Omega} x_{i}^{2}|\nabla v|^{2}+\int_{\Omega} v^{2} .
$$

Substituting (4.51) and (4.52) into (4.50), we get

$$
\Lambda_{i+1}-\Lambda_{1} \leq \frac{-\Lambda_{1} \int_{\Omega} v^{2}+2 l(-1)^{l} \int_{\Omega} x_{i} v\left(\Delta^{l-1} v\right)_{x_{i}}}{\int_{\Omega} x_{i}^{2}|\nabla v|^{2}} .
$$

Since

$$
\Delta^{l-1}\left(x_{i} v\right)=2(l-1)\left(\Delta^{l-2} v\right)_{x_{i}}+x_{i} \Delta^{l-1} v,
$$


we have

$$
\begin{aligned}
\int_{\Omega} x_{i} v\left(\Delta^{l-1} v\right)_{x_{i}} & =\int_{\Omega} x_{i} v \Delta^{l-1} v_{x_{i}} \\
& =\int_{\Omega} \Delta^{l-1}\left(x_{i} v\right) v_{x_{i}} \\
& =\int_{\Omega}\left(2(l-1)\left(\Delta^{l-2} v\right)_{x_{i}}+x_{i} \Delta^{l-1} v\right) v_{x_{i}} \\
& =\int_{\Omega}\left(-2(l-1) \Delta^{l-2} v v_{x_{i} x_{i}}+x_{i} \Delta^{l-1} v v_{x_{i}}\right) .
\end{aligned}
$$

On the other hand, we have from the divergence theorem that

$$
\int_{\Omega} x_{i} v\left(\Delta^{l-1} v\right)_{x_{i}}=-\int_{\Omega} \Delta^{l-1} v\left(v+x_{i} v_{x_{i}}\right) .
$$

Combining (4.54) and (4.55), we obtain

$$
\begin{aligned}
\int_{\Omega} x_{i} v\left(\Delta^{l-1} v\right)_{x_{i}} & =\int_{\Omega}\left((l-1)\left(\Delta^{l-2} v\right)_{x_{i}} v_{x_{i}}-\frac{1}{2} v \Delta^{l-1} v\right) \\
& =\int_{\Omega}\left(-(l-1)\left(\Delta^{l-2} v\right)_{x_{i} x_{i}} v-\frac{1}{2} v \Delta^{l-1} v\right) .
\end{aligned}
$$

Substituting (4.56) into (4.53), we infer

$$
\Lambda_{i+1}-\Lambda_{1} \leq \frac{-\Lambda_{1} \int_{\Omega} v^{2}+l \int_{\Omega}\left(-2(l-1)\left((-\Delta)^{l-2} v\right)_{x_{i} x_{i}} v+v(-\Delta)^{l-1} v\right)}{\int_{\Omega} x_{i}^{2}|\nabla v|^{2}} .
$$

By using the same arguments as in the proof of (4.37), one deduces

$$
\frac{1+4 \int_{\Omega} v_{x_{i}}^{2}+4\left(\int_{\Omega} v_{x_{i}}^{2}\right)^{2}}{\int_{\Omega} x_{i}^{2}|\nabla v|^{2}} \leq 4 \int_{\Omega}\left|\nabla v_{x_{i}}\right|^{2}
$$

which gives

$$
\frac{1}{\int_{\Omega} x_{i}^{2}|\nabla v|^{2}} \leq 4 \int_{\Omega}\left|\nabla v_{x_{i}}\right|^{2}
$$

and since $\sum_{i=1}^{n} \int_{\Omega} v_{x_{i}}^{2}=\int_{\Omega}|\nabla v|^{2}=1$, we know that there exists at least one $i \in\{1, \cdots, n\}$ such that (4.58) is a strict inequality. Set

$$
a=\int_{\Omega} v(-\Delta)^{l-1} v, \quad a_{i}=\int_{\Omega}\left(-\left((-\Delta)^{l-2} v\right)_{x_{i} x_{i}} v\right), i=1, \cdots, n ;
$$

then

$$
\sum_{i=1}^{n} a_{i}=a
$$

Introducing (4.59) into (4.57), we have

$$
\Lambda_{i+1}-\Lambda_{1} \leq 4\left(-\Lambda_{1} \int_{\Omega} v^{2}+l\left(2(l-1) a_{i}+a\right)\right) \int_{\Omega}\left|\nabla v_{x_{i}}\right|^{2},
$$

and there exists at least $i \in\{1, \cdots, n\}$ such that (4.61) is a strict inequality. Take a permutation $\left\{i_{1}, \cdots, i_{n}\right\}$ of $\{1, \cdots, n\}$ so that

$$
a_{i_{n}} \leq a_{i_{n-1}} \leq \cdots \leq a_{i_{1}}
$$


It then follows from (4.60) and (4.62) that

$$
a_{i_{k}} \leq \frac{1}{k} a, k=1, \cdots, n .
$$

Substituting (4.63) into (4.61), we get

$$
\begin{aligned}
\Lambda_{i_{k}+1}-\Lambda_{1} & \leq 4\left(-\Lambda_{1} \int_{\Omega} v^{2}+l\left(2(l-1) a_{i_{k}}+a\right)\right) \int_{\Omega}\left|\nabla v_{x_{i_{k}}}\right|^{2} \\
& \leq 4\left(-\Lambda_{1} \int_{\Omega} v^{2}+l\left(\frac{2(l-1)}{k}+1\right) a\right) \int_{\Omega}\left|\nabla v_{x_{i_{k}}}\right|^{2}
\end{aligned}
$$

and for some $k \in\{1, \cdots, n\},(4.64)$ is a strict inequality. Before we can finish the proof of Theorem 4.3, let us prove the following inequalities:

$$
\begin{aligned}
& \int_{\Omega} v(-\Delta)^{l-1} v \leq \Lambda_{1}^{(l-2) /(l-1)}, \\
& \int_{\Omega} v \Delta^{2} v \leq \Lambda_{1}^{1 /(l-1)}, \\
& \int_{\Omega} v^{2} \geq \Lambda_{1}^{-1 /(l-1)} .
\end{aligned}
$$

First observe as in the proof of Lemma 2.1 that for any $k=1, \cdots, l-1$,

$$
\int_{\Omega} v(-\Delta)^{k} v \geq 0
$$

When $l=2$, (4.65) and (4.66) hold obviously and in this case we have from the Schwarz inequality that

$$
1=\int_{\Omega}(-v \Delta v)=\left(\int_{\Omega}(-v \Delta v)\right)^{2} \leq\left(\int_{\Omega} v^{2}\right)\left(\int_{\Omega}(\Delta v)^{2}\right)=\Lambda_{1} \int_{\Omega} v^{2} .
$$

Hence (4.67) holds when $l=2$.

Assume now that $l>2$. We claim that for any $k=2, \cdots, l-1$,

$$
\left(\int_{\Omega} v(-\Delta)^{k} v\right)^{k} \leq\left(\int_{\Omega} v(-\Delta)^{k+1} v\right)^{k-1} .
$$

Since

$$
\int_{\Omega} v \Delta^{2} v=\int_{\Omega} \Delta v \Delta v=-\int_{\Omega} \nabla \Delta v \nabla v
$$

we have from the Schwarz inequality that

$$
\left(\int_{\Omega} v \Delta^{2} v\right)^{2} \leq\left(\int_{\Omega}|\nabla \Delta v|^{2}\right)\left(\int_{\Omega}|\nabla v|^{2}\right)=-\int_{\Omega} \Delta v \Delta^{2} v=\int_{\Omega} v\left(-\Delta^{3} v\right)
$$

Hence (4.68) holds when $k=2$. Suppose that (4.68) holds for $k-1$, that is,

$$
\left(\int_{\Omega} v(-\Delta)^{k-1} v\right)^{k-1} \leq\left(\int_{\Omega} v(-\Delta)^{k} v\right)^{k-2}
$$


As in the proof of $(2.26)$, we have

$$
\int_{\Omega} v(-\Delta)^{k} v \leq\left(\int_{\Omega} v(-\Delta)^{k-1} v\right)^{1 / 2}\left(\int_{\Omega} v(-\Delta)^{k+1} v\right)^{1 / 2} .
$$

Substituting (4.70) into (4.71), we know that (4.68) is true for $k$. Using (4.68) repeatedly, we get

$$
\begin{gathered}
\int_{\Omega} v(-\Delta)^{k} v \leq\left(\int_{\Omega} v(-\Delta)^{k+1} v\right)^{(k-1) / k} \leq \cdots \\
\leq\left(\int_{\Omega} v(-\Delta)^{l} v\right)^{(k-1) /(l-1)}=\Lambda_{1}^{(k-1) /(l-1)} .
\end{gathered}
$$

Taking $k=2$ and $k=l-1$ in the above inequality, respectively, one gets (4.65) and (4.66). On the other hand, we have from the Schwarz inequality and (4.66) that

$$
\begin{aligned}
1 & =\int_{\Omega}(-v \Delta v)=\left(\int_{\Omega}(-v \Delta v)\right)^{2} \leq\left(\int_{\Omega} v^{2}\right)\left(\int_{\Omega}(\Delta v)^{2}\right) \\
& =\left(\int_{\Omega} v^{2}\right)\left(\int_{\Omega} v \Delta^{2} v\right) \leq \Lambda_{1}^{1 /(l-1)}\left(\int_{\Omega} v^{2}\right) .
\end{aligned}
$$

This proves (4.67). Now we continue with the proof of Theorem 4.3. Substituting (4.65) and (4.67) into (4.64) and multiplying both sides by $\frac{k}{k+2 l}$, we get

$$
\frac{k}{k+2 l}\left(\Lambda_{i_{k}+1}-\Lambda_{1}\right) \leq 4(l-1) \Lambda_{1}^{(l-2) /(l-1)} \int_{\Omega}\left|\nabla v_{x_{i_{k}}}\right|^{2}
$$

and for some $k \in\{1, \cdots, n\}$, the above inequality is a strict inequality. Thus by summing on $k$ and using $\int_{\Omega} v \Delta^{2} v \leq \Lambda_{1}^{1 /(l-1)}$, one gets

$$
\begin{aligned}
\sum_{k=1}^{n} \frac{k}{2 l+k}\left(\Lambda_{i_{k}+1}-\Lambda_{1}\right) & <4(l-1) \Lambda_{1}^{(l-2) /(l-1)} \sum_{k=1}^{n} \int_{\Omega}\left|\nabla v_{x_{i_{k}}}\right|^{2} \\
& =4(l-1) \Lambda_{1}^{(l-2) /(l-1)} \sum_{k=1}^{n} \int_{\Omega}\left|\nabla v_{x_{k}}\right|^{2} \\
& =4(l-1) \Lambda_{1}^{(l-2) /(l-1)} \int_{\Omega} v \Delta^{2} v \leq 4(l-1) \Lambda_{1} .
\end{aligned}
$$

Since $\left\{\frac{k}{2 l+k}\right\}_{k=1}^{n}$ and $\left\{\Lambda_{k+1}-\Lambda_{1}\right\}_{k=1}^{n}$ are two increasing sequences, we have from Lemma 2.5 that

$$
\sum_{k=1}^{n} \frac{k}{2 l+k}\left(\Lambda_{i_{k}+1}-\Lambda_{1}\right) \geq \sum_{k=1}^{n} \frac{k}{2 l+k}\left(\Lambda_{n+2-k}-\Lambda_{1}\right) .
$$

Substituting (4.75) into (4.74), we get (4.47). This completes the proof of Theorem 4.3 . 


\section{EigenVAlues of the POlyHARMONIC OPERATORS ON COMPACT DOMAINS}

\section{IN A UNIT SPHERE}

In this section, we will prove universal inequalities for eigenvalues of the polyharmonic operators on compact connected domains in a unit $n$-sphere $S^{n}$. Let $l$ be

a positive integer and for $p=0,1,2, \ldots$, define the polynomials $F_{p}(t)$ inductively by

$$
\begin{aligned}
& F_{0}(t)=1, \quad F_{1}(t)=t-n, \\
& F_{p}(t)=(2 t-2) F_{p-1}(t)-\left(t^{2}+2 t-n(n-2)\right) F_{p-2}(t), \quad p=2, \cdots .
\end{aligned}
$$

Set

$$
F_{l}(t)=t^{l}+a_{l-1} t^{l-1}+\cdots+a_{1} t+a_{0} .
$$

Theorem 5.1. Let $\lambda_{i}$ be the $i$-th eigenvalue of the following eigenvalue problem:

$$
\begin{aligned}
& (-\Delta)^{l} u=\lambda u \quad \text { in } \Omega \\
& \left.u\right|_{\partial \Omega}=\left.\frac{\partial u}{\partial \nu}\right|_{\partial \Omega}=\cdots=\left.\frac{\partial^{l-1} u}{\partial \nu^{l-1}}\right|_{\partial \Omega}=0
\end{aligned}
$$

where $\Omega$ is a compact connected domain in a unit $n$-sphere $S^{n}$. Then we have

$$
\begin{aligned}
& \sum_{i=1}^{k}\left(\lambda_{k+1}-\lambda_{i}\right)^{2} \\
\leq & \frac{1}{n}\left\{\sum_{i=1}^{k}\left(\lambda_{k+1}-\lambda_{i}\right)^{2}\left(\left|a_{l-1}\right| \lambda_{i}^{(l-1) / l}+\cdots+\left|a_{1}\right| \lambda_{i}^{1 / l}+\left|a_{0}\right|\right)\right\}^{1 / 2} \\
& \times\left\{\sum_{i=1}^{k}\left(\lambda_{k+1}-\lambda_{i}\right)\left(n^{2}+4 \lambda_{i}^{1 / 2}\right)\right\}^{1 / 2} .
\end{aligned}
$$

Corollary 5.1. Under the same assumptions as in Theorem 5.1, we have

$$
\begin{aligned}
\lambda_{k+1} \leq & \frac{1}{k} \sum_{i=1}^{k} \lambda_{i}+\frac{1}{2 n^{2} k^{2}}\left(\sum_{i=1}^{k}\left(\left|a_{l-1}\right| \lambda_{i}^{(l-1) / l}+\cdots+\left|a_{1}\right| \lambda_{i}^{1 / l}+\left|a_{0}\right|\right)\right) \\
& \times\left(k n^{2}+4 \sum_{i=1}^{k} \lambda_{i}^{1 / l}\right) \\
+ & \left\{\frac{1}{4 n^{4} k^{4}}\left(\sum_{i=1}^{k}\left(\left|a_{l-1}\right| \lambda_{i}^{(l-1) l}+\cdots+\left|a_{1}\right| \lambda_{i}^{1 / l}+\left|a_{0}\right|\right)\right)^{2}\left(k n^{2}+4 \sum_{i=1}^{k} \lambda_{i}^{1 / l}\right)^{2}\right. \\
- & \left.\frac{1}{k} \sum_{i=1}^{k}\left(\lambda_{i}-\frac{1}{k} \sum_{j=1}^{k} \lambda_{j}\right)^{2}\right\}^{1 / 2}
\end{aligned}
$$

and

$$
\lambda_{k+1} \leq U_{k+1}+\sqrt{U_{k+1}^{2}-V_{k+1}},
$$


where

$$
U_{k+1}=\frac{1}{k} \sum_{i=1}^{k} \lambda_{i}+\frac{1}{2 n^{2} k} \sum_{i=1}^{k}\left(\left|a_{l-1}\right| \lambda_{i}^{(l-1) / l}+\cdots+\left|a_{1}\right| \lambda_{i}^{1 / l}+\left|a_{0}\right|\right)\left(n^{2}+4 \lambda_{i}^{1 / l}\right)
$$

and

$$
V_{k+1}=\frac{1}{k} \sum_{i=1}^{k} \lambda_{i}^{2}+\frac{1}{n^{2} k} \sum_{i=1}^{k} \lambda_{i}\left(\left|a_{l-1}\right| \lambda_{i}^{(l-1) / l}+\cdots+\left|a_{1}\right| \lambda_{i}^{1 / l}+\left|a_{0}\right|\right)\left(n^{2}+4 \lambda_{i}^{1 / l}\right) .
$$

Proof of Theorem 5.1. Let $x_{1}, x_{2}, \cdots, x_{n+1}$ be the standard coordinate functions of the Euclidean space $\mathbb{R}^{n+1}$; then

$$
S^{n}=\left\{\left(x_{1}, \ldots, x_{n+1}\right) \in \mathbb{R}^{n+1} ; \sum_{\alpha=1}^{n+1} x_{\alpha}^{2}=1\right\}
$$

It is well known that

$$
\Delta x_{\alpha}=-n x_{\alpha}, \quad \alpha=1, \cdots, n+1
$$

Let $u_{i}$ be the $i$-th orthonormal eigenfunction corresponding to the eigenvalue $\lambda_{i}$, $i=1,2, \cdots$. For any $\delta>0$, by taking $h=x_{\alpha}$ in (2.4), we have

$$
\begin{aligned}
& \sum_{i=1}^{k}\left(\lambda_{k+1}-\lambda_{i}\right)^{2} \int_{\Omega}\left(-x_{\alpha} u_{i}^{2} \Delta x_{\alpha}-2 x_{\alpha} u_{i}\left\langle\nabla x_{\alpha}, \nabla u_{i}\right\rangle\right) \\
& \leq \delta \sum_{i=1}^{k+1}\left(\lambda_{k+1}-\lambda_{i}\right)^{2} \int_{\Omega} x_{\alpha} u_{i}\left((-\Delta)^{l}\left(x_{\alpha} u_{i}\right)-\lambda_{i} x_{\alpha} u_{i}\right) \\
& \quad+\frac{1}{\delta} \sum_{i=1}^{k}\left(\lambda_{k+1}-\lambda_{i}\right)\left\|\left\langle\nabla x_{\alpha}, \nabla u_{i}\right\rangle+\frac{u_{i} \Delta x_{\alpha}}{2}\right\|^{2} .
\end{aligned}
$$

Taking the sum on $\alpha$ from 1 to $n+1$, we get

$$
\begin{aligned}
\sum_{i=1}^{k}\left(\lambda_{k+1}-\right. & \left.\lambda_{i}\right)^{2} \sum_{\alpha=1}^{n+1} \int_{\Omega}\left(-x_{\alpha} u_{i}^{2} \Delta x_{\alpha}-2 x_{\alpha} u_{i}\left\langle\nabla x_{\alpha}, \nabla u_{i}\right\rangle\right) \\
\leq & \delta \sum_{i=1}^{k+1}\left(\lambda_{k+1}-\lambda_{i}\right)^{2} \sum_{\alpha=1}^{n+1} \int_{\Omega} x_{\alpha} u_{i}\left((-\Delta)^{l}\left(x_{\alpha} u_{i}\right)-\lambda_{i} x_{\alpha} u_{i}\right) \\
& +\frac{1}{\delta} \sum_{i=1}^{k}\left(\lambda_{k+1}-\lambda_{i}\right) \sum_{\alpha=1}^{n+1}\left\|\left\langle\nabla x_{\alpha}, \nabla u_{i}\right\rangle+\frac{u_{i} \Delta x_{\alpha}}{2}\right\|^{2}
\end{aligned}
$$


Using $\sum_{\alpha=1}^{n+1} x_{\alpha}^{2}=1,(2.17)$ and (5.8), we infer

$$
\begin{aligned}
& \sum_{\alpha=1}^{n+1} \int_{\Omega}\left(-x_{\alpha} u_{i}^{2} \Delta x_{\alpha}-2 x_{\alpha} u_{i}\left\langle\nabla x_{\alpha}, \nabla u_{i}\right\rangle\right) \\
& \quad=\int_{\Omega}\left(\left(\sum_{\alpha=1}^{n+1} x_{\alpha}^{2}\right) n u_{i}^{2}-u_{i}\left\langle\nabla\left(\sum_{\alpha=1}^{n+1} x_{\alpha}^{2}\right), \nabla u_{i}\right\rangle\right)=\int_{\Omega} n u_{i}^{2}=n \\
& \sum_{\alpha=1}^{n+1} \|\left\langle\left\langle x_{\alpha}, \nabla u_{i}\right\rangle+\frac{u_{i} \Delta x_{\alpha}}{2} \|^{2}\right. \\
& \quad=\int_{\Omega} \sum_{\alpha=1}^{n+1}\left(\left\langle\nabla x_{\alpha}, \nabla u_{i}\right\rangle^{2}-n\left\langle\nabla x_{\alpha}, \nabla u_{i}\right\rangle u_{i} x_{\alpha}+\frac{n^{2} u_{i}^{2} x_{\alpha}^{2}}{4}\right) \\
& \quad=\frac{n^{2}}{4}+\int_{\Omega}\left|\nabla u_{i}\right|^{2} \\
& \quad=\frac{n^{2}}{4}+\int_{\Omega} u_{i}\left(-\Delta u_{i}\right) \\
& \leq \frac{n^{2}}{4}+\lambda_{i}^{1 / l} .
\end{aligned}
$$

For any smooth function $f$ on $\Omega$, we have from the Bochner formula that

$$
\begin{aligned}
\frac{1}{2} \Delta|\nabla f|^{2} & =\left|\nabla^{2} f\right|^{2}+\langle\nabla f, \nabla(\Delta f)\rangle+\operatorname{Ric}(\nabla f, \nabla f) \\
& =\left|\nabla^{2} f\right|^{2}+\langle\nabla f, \nabla(\Delta f)\rangle+(n-1)|\nabla f|^{2},
\end{aligned}
$$

where Ric is the Ricci tensor of $S^{n}$. Thus for any smooth function $g$ on $\Omega$,

$$
\frac{1}{2} \Delta|\nabla g|^{2}=\left|\nabla^{2} g\right|^{2}+\langle\nabla g, \nabla(\Delta g)\rangle+(n-1)|\nabla g|^{2}
$$

and

$$
\frac{1}{2} \Delta|\nabla(f+g)|^{2}=\left|\nabla^{2}(f+g)\right|^{2}+\langle\nabla(f+g), \nabla(\Delta(f+g))\rangle+(n-1)|\nabla(f+g)|^{2} .
$$

Subtracting the sum of (5.12) and (5.13) from (5.14), we get

$$
\Delta\langle\nabla f, \nabla g\rangle=2\left\langle\nabla^{2} f, \nabla^{2} g\right\rangle+\langle\nabla f, \nabla(\Delta g)\rangle+\langle\nabla g, \nabla(\Delta f)\rangle+2(n-1)\langle\nabla f, \nabla g\rangle,
$$

where

$$
\left\langle\nabla^{2} f, \nabla^{2} g\right\rangle=\sum_{s, t=1}^{n} \nabla^{2} f\left(e_{s}, e_{t}\right) \nabla^{2} g\left(e_{s}, e_{t}\right),
$$

$e_{1}, \cdots, e_{n}$ being orthonormal vector fields locally defined on $\Omega$. Since

$$
\nabla^{2} x_{\alpha}=-x_{\alpha}\langle,\rangle,
$$

we infer from (5.15) by taking $f=x_{\alpha}$ that

$$
\begin{aligned}
\Delta\left\langle\nabla x_{\alpha}, \nabla g\right\rangle & =-2 x_{\alpha} \Delta g+\left\langle\nabla x_{\alpha}, \nabla(\Delta g)\right\rangle+(n-2)\left\langle\nabla x_{\alpha}, \nabla g\right\rangle \\
& =-2 x_{\alpha} \Delta g+\left\langle\nabla x_{\alpha}, \nabla((\Delta+(n-2)) g)\right\rangle .
\end{aligned}
$$

For each $q=0,1, \cdots$, thanks to (5.8) and (5.16), there are polynomials $B_{q}$ and $C_{q}$ of degrees less than or equal to $q$ such that

$$
\Delta^{q}\left(x_{\alpha} g\right)=x_{\alpha} B_{q}(\Delta) g+2\left\langle\nabla x_{\alpha}, \nabla\left(C_{q}(\Delta) g\right)\right\rangle .
$$


It is obvious that

$$
B_{0}=1, \quad B_{1}=t-n, \quad C_{0}=0, \quad C_{1}=1 .
$$

It follows from (5.8), (5.16) and (5.17) that

$$
\begin{aligned}
\Delta^{q}\left(x_{\alpha} g\right)= & \Delta\left(\Delta^{q-1}\left(x_{\alpha} g\right)\right) \\
= & \Delta\left(x_{\alpha} B_{q-1}(\Delta) g+2\left\langle\nabla x_{\alpha}, \nabla\left(C_{q-1}(\Delta) g\right)\right\rangle\right) \\
= & x_{\alpha}\left((\Delta-n) B_{q-1}(\Delta)-4 \Delta C_{q-1}(\Delta)\right) g \\
& \quad+2\left\langle\nabla x_{\alpha}, \nabla\left(\left(B_{q-1}(\Delta)+(\Delta+(n-2)) C_{q-1}(\Delta)\right) g\right)\right\rangle .
\end{aligned}
$$

Thus, for any $q=2, \cdots$, we have

$$
\begin{aligned}
& B_{q}(\Delta)=(\Delta-n) B_{q-1}(\Delta)-4 \Delta C_{q-1}(\Delta), \\
& C_{q}(\Delta)=B_{q-1}(\Delta)+(\Delta+(n-2)) C_{q-1}(\Delta) .
\end{aligned}
$$

Consequently, we have

$$
\begin{aligned}
B_{q}(\Delta)= & (2 \Delta-2) B_{q-1}(\Delta)-(\Delta+n-2) B_{q-1}(\Delta)-4 \Delta C_{q-1}(\Delta) \\
= & (2 \Delta-2) B_{q-1}(\Delta)-(\Delta+n-2)\left((\Delta-n) B_{q-2}(\Delta)-4 \Delta C_{q-2}(\Delta)\right) \\
& -4 \Delta C_{q-1}(\Delta) \\
= & (2 \Delta-2) B_{q-1}(\Delta)-\left(\Delta^{2}+2 \Delta-n(n-2)\right) B_{q-2}(\Delta) \\
& +4 \Delta\left[B_{q-2}(\Delta)+(\Delta+n-2) C_{q-2}(\Delta)-C_{q-1}(\Delta)\right] \\
= & (2 \Delta-2) B_{q-1}(\Delta)-\left(\Delta^{2}+2 \Delta-n(n-2)\right) B_{q-2}(\Delta), \quad q=2, \cdots .
\end{aligned}
$$

Since (5.18) and (5.22) hold, we know that $B_{q}=F_{q}, \forall q=0,1, \cdots$. It follows from (5.17) and the divergence theorem that

$$
\begin{aligned}
& \int_{\Omega} x_{\alpha} u_{i}\left((-\Delta)^{l}\left(x_{\alpha} u_{i}\right)-\lambda_{i} x_{\alpha} u_{i}\right) \\
= & \int_{\Omega} x_{\alpha} u_{i}\left((-1)^{l}\left(x_{\alpha} B_{l}(\Delta) u_{i}+2\left\langle\nabla x_{\alpha}, \nabla\left(C_{l}(\Delta) u_{i}\right)\right\rangle\right)-\lambda_{i} x_{\alpha} u_{i}\right) \\
= & \int_{\Omega} x_{\alpha} u_{i}\left(( - 1 ) ^ { l } \left(x_{\alpha}\left(\Delta^{l}+a_{l-1} \Delta^{l-1}+\cdots+a_{0}\right) u_{i}\right.\right. \\
& \left.\left.+2\left\langle\nabla x_{\alpha}, \nabla\left(C_{l}(\Delta) u_{i}\right)\right\rangle\right)-\lambda_{i} x_{\alpha} u_{i}\right) \\
= & \int_{\Omega}(-1)^{l} x_{\alpha} u_{i}\left(x_{\alpha}\left(a_{l-1} \Delta^{l-1}+\cdots+a_{0}\right) u_{i}+2\left\langle\nabla x_{\alpha}, \nabla\left(C_{l}(\Delta) u_{i}\right)\right\rangle\right) .
\end{aligned}
$$

Summing on $\alpha$, one has from $\nabla\left(\sum_{\alpha=1}^{n+1} x_{\alpha}^{2}\right)=0$ and (2.17) that

$$
\begin{aligned}
& \int_{\Omega} x_{\alpha} u_{i}\left((-\Delta)^{l}\left(x_{\alpha} u_{i}\right)-\lambda_{i} x_{\alpha} u_{i}\right) \\
= & \int_{\Omega} u_{i}(-1)^{l}\left(a_{l-1} \Delta^{l-1}+\cdots+a_{0}\right) u_{i} \\
\leq & \left|a_{l-1}\right| \int_{\Omega} u_{i}(-\Delta)^{l-1} u_{i}+\cdots+\left|a_{1}\right| \int_{\Omega} u_{i}(-\Delta) u_{i}+\left|a_{0}\right| \int_{\Omega} u_{i}^{2} \\
\leq & \left|a_{l-1}\right| \lambda_{i}^{(l-1) / l}+\cdots+\left|a_{1}\right| \lambda_{i}^{1 / l}+\left|a_{0}\right| .
\end{aligned}
$$


Substituting (5.10), (5.11) and (5.24) into (5.9), we infer

$$
\begin{aligned}
n \sum_{i=1}^{k}\left(\lambda_{k+1}-\lambda_{i}\right)^{2} \leq & \delta \sum_{i=1}^{k}\left(\lambda_{k+1}-\lambda_{i}\right)^{2}\left(\left|a_{l-1}\right| \lambda_{i}^{(l-1) / l}+\cdots+\left|a_{1}\right| \lambda_{i}^{1 / l}+\left|a_{0}\right|\right) \\
& +\frac{1}{\delta} \sum_{i=1}^{k}\left(\lambda_{k+1}-\lambda_{i}\right)\left(\lambda_{i}^{1 / l}+\frac{n^{2}}{4}\right) .
\end{aligned}
$$

Taking

$$
\delta=\left\{\frac{\sum_{i=1}^{k}\left(\lambda_{k+1}-\lambda_{i}\right)\left(\lambda_{i}^{1 / l}+\frac{n^{2}}{4}\right)}{\sum_{i=1}^{k}\left(\lambda_{k+1}-\lambda_{i}\right)^{2}\left(\left|a_{l-1}\right| \lambda_{i}^{(l-1) / l}+\cdots+\left|a_{1}\right| \lambda_{i}^{1 / l}+\left|a_{0}\right|\right)}\right\}^{1 / 2},
$$

we get (5.1). This completes the proof of Theorem 5.1.

Proof of Corollary 5.1. From Lemma 2.4, we have

$$
\begin{aligned}
& \sum_{i=1}^{k}\left(\lambda_{k+1}-\lambda_{i}\right)^{2}\left(\left|a_{l-1}\right| \lambda_{i}^{(l-1) / l}+\cdots+\left|a_{1}\right| \lambda_{i}^{1 / l}+\left|a_{0}\right|\right) \\
\leq & \frac{1}{k}\left\{\sum_{i=1}^{k}\left(\lambda_{k+1}-\lambda_{i}\right)^{2}\right\}\left\{\sum_{i=1}^{k}\left(\left|a_{l-1}\right| \lambda_{i}^{(l-1) / l}+\cdots+\left|a_{1}\right| \lambda_{i}^{1 / l}+\left|a_{0}\right|\right)\right\}
\end{aligned}
$$

and

$$
\begin{aligned}
\sum_{i=1}^{k}\left(\lambda_{k+1}-\lambda_{i}\right)\left(n^{2}+4 \lambda_{i}^{1 / l}\right) & \leq \frac{1}{k}\left\{\sum_{i=1}^{k}\left(\lambda_{k+1}-\lambda_{i}\right)\right\}\left\{\sum_{i=1}^{k}\left(n^{2}+4 \lambda_{i}^{1 / l}\right)\right\} \\
& =\frac{1}{k}\left\{\sum_{i=1}^{k}\left(\lambda_{k+1}-\lambda_{i}\right)\right\}\left\{k n^{2}+4 \sum_{i=1}^{k} \lambda_{i}^{1 / l}\right\}
\end{aligned}
$$

Substituting (5.26) and (5.27) into (5.3), we get

$$
\begin{aligned}
\sum_{i=1}^{k}\left(\lambda_{k+1}-\lambda_{i}\right)^{2} \leq \frac{1}{n^{2} k^{2}}\left\{\sum_{i=1}^{k}\right. & \left.\left(\left|a_{l-1}\right| \lambda_{i}^{(l-1) / l}+\cdots+\left|a_{1}\right| \lambda_{i}^{1 / l}+\left|a_{0}\right|\right)\right\} \\
& \times\left\{\sum_{i=1}^{k}\left(\lambda_{k+1}-\lambda_{i}\right)\right\}\left\{k n^{2}+4 \sum_{i=1}^{k} \lambda_{i}^{1 / l}\right\} .
\end{aligned}
$$

Solving this quadratic polynomial of $\lambda_{k+1}$, we have (5.4).

On the other hand, one gets by using (2.27) that

$$
\begin{aligned}
& \left\{\sum_{i=1}^{k}\left(\lambda_{k+1}-\lambda_{i}\right)^{2}\left(\left|a_{l-1}\right| \lambda_{i}^{(l-1) / l}+\cdots+\left|a_{1}\right| \lambda_{i}^{1 / l}+\left|a_{0}\right|\right)\right\} \\
& \quad \times\left\{\sum_{i=1}^{k}\left(\lambda_{k+1}-\lambda_{i}\right)\left(n^{2}+4 \lambda_{i}^{1 / 2}\right)\right\} \leq\left\{\sum_{i=1}^{k}\left(\lambda_{k+1}-\lambda_{i}\right)^{2}\right\} \\
& \quad \times\left\{\sum_{i=1}^{k}\left(\lambda_{k+1}-\lambda_{i}\right)\left(\left|a_{l-1}\right| \lambda_{i}^{(l-1) / l}+\cdots+\left|a_{1}\right| \lambda_{i}^{1 / l}+\left|a_{0}\right|\right)\left(n^{2}+4 \lambda_{i}^{1 / 2}\right)\right\}
\end{aligned}
$$


which, combining with (5.3), gives

$$
\begin{aligned}
& \sum_{i=1}^{k}\left(\lambda_{k+1}-\lambda_{i}\right)^{2} \\
& \leq \frac{1}{n^{2}} \sum_{i=1}^{k}\left(\lambda_{k+1}-\lambda_{i}\right)\left(\left|a_{l-1}\right| \lambda_{i}^{(l-1) / l}+\cdots+\left|a_{1}\right| \lambda_{i}^{1 / l}+\left|a_{0}\right|\right)\left(n^{2}+4 \lambda_{i}^{1 / l}\right) .
\end{aligned}
$$

Hence

$$
\lambda_{k+1} \leq U_{k+1}+\sqrt{U_{k+1}^{2}-V_{k+1}},
$$

where $U_{k+1}$ and $V_{k+1}$ are given by (5.6) and (5.7), respectively. Thus (5.5) holds.

\section{ACKNOWLEDGEMENTS}

The authors are very grateful to the referee for numerous valuable comments and suggestions. The fourth author also thanks the Max Planck Institute for Mathematics in the Sciences for its hospitality during the preparation of this paper and CAPES.

\section{REFERENCES}

[A1] M. S. Ashbaugh, Isoperimetric and universal inequalities for eigenvalues, in Spectral theory and geometry (Edinburgh, 1998), E. B. Davies and Yu Safalov, eds., London Math. Soc. Lecture Notes, vol. 273, Cambridge Univ. Press, Cambridge, 1999, pp. 95139. MR1736867 (2001a:35131)

[A2] M. S. Ashbaugh, The universal eigenvalue bounds of Payne-Pólya-Weinberger, HileProtter, and H. C. Yang. Spectral and inverse spectral theory (Goa, 2000). Proc. Indian Acad. Sci. Math. Sci. 112 (2002), 3-30. MR1894540 (2004c:35302)

[AB1] M. S. Ashbaugh and R. D. Benguria, Proof of the Payne-Pólya-Weinberger conjecture, Bull. Amer. Math. Soc. 25 (1991), 19-29. MR1085824 (91m:35173)

[AB2] M. S. Ashbaugh and R. D. Benguria, A sharp bound for the ratio of the first two eigenvalues of Dirichlet Laplacians and extensions, Ann. of Math. (2) 135 (1992), 601-628. MR.1166646 (93d:35105)

[AB3] M. S. Ashbaugh and R. D. Benguria, A second proof of the Payne-Pólya-Weinberger conjecture, Commun. Math. Phys. 147 (1992), 181-190. MR.1171765 (93k:33002)

[AB4] M. S. Ashbaugh and R. D. Benguria, More bounds on eigenvalue ratios for Dirichlet Laplacians in $n$ dimensions, SIAM J. Math. Anal. 24 (1993), 1622-1651. MR1241161 (94i:35139)

[AH] M. S. Ashbaugh and L. Hermi, A unified approach to universal inequalities for eigenvalues of elliptic operators, Pacific J. Math. 217 (2004), 201-219. MR2109931 (2005k:35305)

[Ch] I. Chavel, Eigenvalues in Riemannian geometry, Academic Press, 1984. MR768584 (86g:58140)

[CIM] Q. M. Cheng, T. Ichikawa, and S. Mametsuka, Estimates for eigenvalues of Laplacian with any order in a unit sphere, Calc. Var. PDE 36 (2009), 507-523. MR 2558327 (2010k:35332)

[CY1] Q. M. Cheng and H. C. Yang, Estimates on eigenvalues of Laplacian. Math. Ann. 331 (2005),445-460. MR2115463(2005i:58038)

[CY2] Q. M. Cheng and H. C. Yang, Universal bounds for eigenvalues of a buckling problem, Comm. Math. Phys. 262 (2006), 663-675. MR2202307 (2007f:35056)

[CY3] Q. M. Cheng and H. C. Yang, Inequalities for eigenvalues of a clamped plate problem, Trans. Amer. Math. Soc., 358 (2006), 2625-2635. MR2204047 (2006m:35263)

[CY4] Q. M. Cheng and H. C. Yang, Inequalities for eigenvalues of Laplacian on domains and compact complex hypersurfaces in complex projective spaces, J. Math. Soc. Japan, 58 (2006), 545-561. MR2228572(2007k:58051)

[CQ] Z. C. Chen and C. L. Qian, Estimates for discrete spectrum of Laplacian operator with any order, J. China Univ. Sci. Tech. 20 (1990), 259-266. MR.1077287 (92c:35087) 
[Co] R. Courant, Über die Schwingungen eingespannter Platten, Math. Zeitschr. 15 (1922), 195-200. MR1544567

[CH] R. Courant and D. Hilbert, Methoden der mathematischen Physik I, 3rd ed., Springer, 1968. MR0344038 (49:8778)

[HLP] G. Hardy, J. E. Littlewood and G. Pólya, Inequalities, Reprint of the 1952 edition, Cambridge University Press, 1988. MR0944909 (89d:26016)

[Ha] E. M. Harrell, Some geometric bounds on eigenvalue gaps, Commun. Part. Differ. Equ. 18 (1993), 179-198. MR.1211730 (94c:35135)

[HM1] E. M. Harrell and P. L. Michel, Commutator bounds for eigenvalues, with applications to spectral geometry, Commun. Part. Differ. Equ. 19 (1994), 2037-2055. MR 1301181 (95i:58182)

[HM2] E. M. Harrell and P. L. Michel, Commutator bounds for eigenvalues of some differential operators, Lecture Notes in Pure and Applied Mathematics, vol. 168 (G. Ferreyra, G. R. Goldstein and F. Neubrander, eds.) (New York: Marcel Dekker) (1995) pp. 235-244. MR.1300432 (95j:47028)

[HS] E. M. Harrell and J. Stubbe, On trace inequalities and the universal eigenvalue estimates for some partial differential operators, Trans. Amer. Math. Soc. 349 (1997), 1797-1809. MR.1401772 (97i:35129)

[HP] G. N. Hile and M. H. Protter, Inequalities for eigenvalues of the Laplacian, Indiana Univ. Math. J. 29 (1980), 523-538. MR578204 (82c:35052)

[HY] G. N. Hile and R. Z. Yeh, Inequalities for eigenvalues of the biharmonic operator, Pacific J. Math. 112 (1984), 115-133. MR739143 (85k:35170)

$[\mathrm{H}] \quad$ S. M. Hook, Domain-independent upper bounds for eigenvalues of elliptic operators, Trans. Amer. Math. Soc. 318 (1990), 615-642. MR.994167(90h:35075)

[LeP] M. Levitin and L. Parnovski, Commutators, spectral trace identities and universal estimates for eigenvalues, J. Funct. Anal. 192 (2002), 425-445. MR.1923409 (2003g:47040)

[PPW1] L. E. Payne, G. Pólya and H. F. Weinberger, Inequalities for eigenvalues of plates and membranes, J. Rational Mech. Anal. 4 (1955), 517-529. MR0070834 (17:42a)

[PPW2] L. E. Payne, G. Pólya and H. F. Weinberger, On the ratio of consecutive eigenvalues, J. Math. and Phys. 35 (1956), 289-298. MR0084696 (18:905c)

[WX1] Q. Wang and C. Xia, Universal bounds for eigenvalues of the biharmonic operator on Riemannian manifolds, J. Funct. Anal. 245 (2007), 334-352. MR2311628 (2008e:58033)

[WX2] Q. Wang and C. Xia, Universal inequalities for eigenvalues of the buckling problem on spherical domains, Comm. Math. Phys. 270 (2007), 759-775. MR2276464(2007m:35180)

[We] H. Weyl, Das asymptotische Verteilungsgesetz der Eigenwerte linearer partieller Differentialgleichungen, Math. Ann. 71 (1912), 441-469. MR1511670

[WC] F. E. Wu and L. F. Cao, Estimates for eigenvalues of Laplacian operator with any order, Science in China A: Mathematics 50 (2007), 1078-1086. MR.2370015 (2009c:35076)

[Y] H. C. Yang, Estimates of the difference between consecutive eigenvalues, preprint, 1995 (revision of International Centre for Theoretical Physics preprint IC/91/60, Trieste, Italy, April 1991).

Max Planck Institute for Mathematics in the Sciences, 04103 Leipzig, Germany

E-mail address: jost@mis.mpg.de

Max Planck Institute for Mathematics in the Sciences, 04103 Leipzig, Germany

E-mail address: xli-jost@mis.mpg.de

Departamento de Matemática, University of Brasilia, 70910-900, Brasília-DF, Brazil E-mail address: wang@mat.unb.br

Departamento de Matemática, University of Brasilia, 70910-900, Brasília-DF, Brazil

E-mail address: xia@mat.unb.br 